\title{
Existence and Uniqueness of Positive (Almost) Periodic Solutions for a Neutral Multi-Species Logarithmic Population Model with Multiple Delays and Impulses
}

\author{
Zhenguo Luo ${ }^{1,2 \#}$, Jianhua Huang ${ }^{1}$, Liping Luo $^{2}$, Binxiang Dai ${ }^{3}$ \\ ${ }^{1}$ Department of Mathematics, National University of Defense Technology, Changsha, China \\ ${ }^{2}$ Department of Mathematics, Hengyang Normal University, Hengyang, China \\ ${ }^{3}$ School of Mathematical Sciences and Statistics, Central South University, Changsha, China \\ Email: "luozhenguo0701@yahoo.com.cn
}

Received January 28, 2013; revised March 2, 2013; accepted March 10, 2013

Copyright (C) 2013 Zhenguo Luo et al. This is an open access article distributed under the Creative Commons Attribution License, which permits unrestricted use, distribution, and reproduction in any medium, provided the original work is properly cited.

\begin{abstract}
In this paper, by using the contraction mapping principle and constructing a suitable Lyapunov functional, we established a set of easily applicable criteria for the existence, uniqueness and global attractivity of positive periodic solution and positive almost periodic solution of a neutral multi-species Logarithmic population model with multiple delays and impulses. The results improve and generalize the known ones in [1], as an application, we also give an example to illustrate the feasibility of our main results.
\end{abstract}

Keywords: Contraction Mapping Principle; Impulses; Lyapunov Functional; Global Attractivity; Uniqueness; Positive Periodic Solution; Almost Periodic Solution

\section{Introduction}

Recently, there are more works on the periodic solution of neutral type Logistic models or Lotka-Volterra models (see [2-7] for details). Only a little scholars considered the neutral Logarithmic model (see [1,8-10]). In [8], Li had studied the following single species neutral Logarithmic model:

$$
\begin{aligned}
& N^{\prime}(t)= \\
& N(t)\left[r(t)-a(t) \ln N(t-\sigma)-b(t)(\ln N(t-\eta))^{\prime}\right]^{\prime} .
\end{aligned}
$$

He had established a set of easily applicable criteria for the existence of positive periodic solution of system (1.1) by applying the continuation theorem of the coincidence degree theory which proposed in [11] by Mawhin. In [9], $\mathrm{Lu}$ and $\mathrm{Ge}$ employed an abstract continuous theorem of k-set contractive operator to investigate the following equation:

\footnotetext{
*Supported by NSF of China (No. 10971229, 11161015), PSF of China (2012M512162) and NSF of Hunan Province (No. 11JJ9002, 13JJ4098). \#Corresponding author.
}

$$
\begin{aligned}
N^{\prime}(t)= & N(t)\left[r(t)-\sum_{j=1}^{n} a_{j}(t) \ln N\left(t-\sigma_{j}(t)\right)\right. \\
& \left.-\sum_{i=1}^{m} b_{i}(t)\left(\ln N\left(t-\tau_{i}(t)\right)\right)^{\prime}\right]
\end{aligned}
$$

They established some criteria to guarantee the existence of positive periodic solutions of system (1.2). In [10], Chen studied the following neutral multi-species Logarithmic population model:

$$
\begin{aligned}
\frac{\mathrm{d} N_{i}(t)}{\mathrm{d} t}= & N_{i}(t)\left[r_{i}(t)-\sum_{j=1}^{n} a_{i j}(t) \ln N_{j}(t)\right. \\
& -\sum_{j=1}^{n} b_{i j}(t) \ln N_{j}\left(t-\tau_{i j}(t)\right) \\
& -\sum_{j=1}^{n} c_{i j}(t) \int_{-\infty}^{t} K_{i j}(t-s) \ln N_{j}((s)) \mathrm{d} s \\
& \left.-\sum_{j=1}^{n} d_{i j}(t) \frac{\mathrm{d} \ln N_{j}\left(t-\eta_{i j}(t)\right)}{\mathrm{d} t}\right] .
\end{aligned}
$$


By using the method of fixed point theory and constructing a suitable Lyapunov functional, a set of easily applicable criteria are established for the existence, uniqueness and global attractivity of positive periodic solution (positive almost periodic solution) for system (1.3).

In [1], Wang et al. had investigated the existence, uniqueness of the positive periodic solution of the following neutral multi-species Logarithmic population model:

$$
\begin{aligned}
N^{\prime}(t)= & N(t)[r(t)-a(t) \ln N(t) \\
& -\sum_{j=1}^{n} b_{j}(t) \ln N\left(t-\tau_{j}(t)\right) \\
& -\sum_{j=1}^{n} c_{j}(t) \int_{-\infty}^{t} k_{j}(t-s) \ln N((s)) \mathrm{d} s \\
& \left.-\sum_{j=1}^{n} d_{j}(t)\left(\ln N\left(t-\eta_{j}(t)\right)\right)^{\prime}\right] .
\end{aligned}
$$

By using an abstract continuous theorem of $k$-set contractive operator, the criteria is established for the existence, global attractivity of positive periodic solutions for model (1.4).

On the other hand, there are some other perturbations in the real world such as fires and floods that are not suitable to be considered continually. These perturbations bring sudden changes to the system. Systems with such sudden perturbations involving impulsive differential equations have attracted the interest of many researchers in the past twenty years [12-20], since they provide a natural description of several real processes subject to certain perturbations whose duration is negligible in comparison with the duration of the process. Such processes are often investigated in various fields of science and technology such as physics, population dynamics, ecology, biological systems, optimal control, etc. For details, see $[21,22]$. Recently, the corresponding theory for impulsive functional differential equations has been studied by many authors [23-25]. However there are few published papers discussing the impulsive neutral multispecies Logarithmic population model. Our method is different from that in [1,9].

In this paper, we investigate the existence, uniqueness of the positive periodic solution of the following neutral multi-species Logarithmic population system with multiple delays and impulses

$$
\left\{\begin{aligned}
\frac{\mathrm{d} N_{i}(t)}{\mathrm{d} t}= & N_{i}(t)\left[a_{i}(t)-b_{i}(t) \ln N_{i}(t)-\sum_{j=1}^{n} c_{i j}(t) \ln N_{j}(t)-\sum_{j=1}^{n} d_{i j}(t) \ln N_{j}\left(t-\gamma_{i j}(t)\right)\right. \\
& \left.-\sum_{j=1}^{n} e_{i j}(t) \int_{-\infty}^{t} K_{i j}(t-s) \ln N_{j}(s) \mathrm{d} s-\sum_{j=1}^{n} f_{i j}(t) \frac{\mathrm{d} \ln N_{j}\left(t-\delta_{i j}(t)\right)}{\mathrm{d} t}\right], i=1,2, \cdots, n, t \neq t_{k}, \\
\Delta N_{i}\left(t_{k}\right)= & N_{i}\left(t_{k}^{+}\right)-N_{i}\left(t_{k}\right)=\theta_{i k} N_{i}\left(t_{k}\right), i=1,2, \cdots, n, k=1,2, \cdots .
\end{aligned}\right.
$$

where $i=1,2, \cdots, n, a_{i}(t), b_{i}(t), c_{i j}(t), d_{i j}(t), e_{i j}(t)$, $f_{i j}(t) \in C(R,(0,+\infty)), \quad \gamma_{i j}(t), \quad \delta_{i j}(t) \in C(R,[0,+\infty))$ are all continuous functions with $\gamma=\max \left\{\gamma_{i j}(t), \delta(t)\right\}$, $\gamma_{i j}^{\prime}(t)<1, \quad \delta_{i j}^{\prime}(t)<1$. And $1+\theta_{i k}>0, \int_{0}^{\infty} K_{i j}(s) \mathrm{d} s=1$, $\int_{0}^{\infty} s K_{i j}(s) \mathrm{d} s<+\infty$. We consider (1.5) together with the initial conditions

$$
\begin{aligned}
& N_{i}(\xi)=\phi_{i}(\xi), N_{i}^{\prime}(\xi)=\phi_{i}^{\prime}(\xi), \xi \in[-\tau, 0], \phi_{i}(0)>0, \\
& \phi_{i} \in C([-\gamma, 0),[0,+\infty)) \cap C^{1}([-\gamma, 0),[0,+\infty)), \\
& i=1,2, \cdots, n .
\end{aligned}
$$

For the ecological justification of (1.5) and the similar types refer to [1,8-10].

Throughout this paper, we make the following notations:

Let $\omega>0$ be a constant and

$$
C_{\omega}=\left\{x(t)=\left(x_{1}(t), \cdots, x_{n}(t)\right)^{\mathrm{T}} \mid x_{i}(t) \in C(R, R), x_{i}(t+\omega)=x_{i}(t)\right\},
$$

with the norm defined by $\|x\|_{1}=\max _{t \in[0, \omega]}\left\{\left|x_{i}(t)\right|\right\}$;

$$
C_{\omega}^{1}=\left\{x(t)=\left(x_{1}(t), \cdots, x_{n}(t)\right)^{\mathrm{T}} \mid x_{i}(t) \in C^{1}(R, R), x_{i}(t+\omega)=x_{i}(t)\right\},
$$


with the norm defined by $\|x\|_{2}=\max _{t \in[0, \omega]}\left\{\|x\|_{1},\left\|x^{\prime}\right\|_{1}\right\}$.

Then $\left(C_{\omega},\|x\|_{1}\right),\left(C_{\omega}^{1},\|x\|_{2}^{1}\right)^{1}$ are Banach spaces.

For the sake of generality and convenience, we always make the following fundamental assumptions:

$\left(\mathrm{H}_{1}\right) a_{i}(t), b_{i}(t), c_{i j}(t), d_{i j}(t), e_{i j}(t)$, are all positive periodic continuous functions with period $\omega$, and $f_{i j}(t)$ are positive continuously differentiable $\omega$-periodic functions. Furthermore, $\gamma_{i j}(t), \delta_{i j}(t)$ are positive $\omega$ periodic continuous functions such that $\gamma_{i j}^{\prime}(t)<1$, $\delta_{i j}^{\prime}(t)<1$, and $\delta_{i j}^{\prime \prime}(t)$ exists;

$\left(\mathrm{H}_{2}\right) 0<t_{1}<t_{2}<\cdots<t_{k}<\cdots$ are fixed impulsive points with $\lim _{k \rightarrow \infty} t_{k}=+\infty$;

$\left(\mathrm{H}_{3}\right)\left\{\theta_{i k}\right\}$ is a real sequence such that $\theta_{i k}+1>0$, $\prod_{0<t_{k}<t}\left(1+\theta_{i k}\right)$ is an $\omega$-periodic function;

$\left(\mathrm{H}_{4}\right) a_{i}(t), b_{i}(t), c_{i j}(t), d_{i j}(t), e_{i j}(t)$, are all almost periodic continuous functions with period $\omega$ on $\mathrm{R}$, and $f_{i j}(t)$ are positive continuously differentiable almost periodic functions such that

$$
\begin{gathered}
a_{i}(t) \geq 0, b_{i}(t) \geq 0, c_{i j}(t) \geq 0, d_{i j}(t) \geq 0, e_{i j}(t) \geq 0, \\
f_{i j}(t) \geq 0, m\left(b_{i}(t)+d_{i i}(t)\right)>0,
\end{gathered}
$$

where

$$
\begin{aligned}
& m\left(b_{i}(t)+d_{i i}(t)\right)=\lim _{T \rightarrow+\infty} \frac{1}{T} \int_{t}^{t+T}\left(b_{i}(\tau)+d_{i i}(\tau)\right) \mathrm{d} \tau, \\
& i, j=1,2, \cdots, n ;
\end{aligned}
$$

$\left(\mathrm{H}_{5}\right) \gamma_{i j}(t), \delta_{i j}(t)$ are positive continuously differentiable almost periodic functions such that $\gamma_{i j}^{\prime}(t)<1$, $\delta_{i j}^{\prime}(t)<1$, and $\delta_{i j}^{\prime \prime}(t)$ exists, $0<t_{1}<t_{2}<\cdots<t_{k}<\cdots$ are fixed impulsive points with $\lim _{k \rightarrow \infty} t_{k}=+\infty$;

$\left(\mathrm{H}_{6}\right)\left\{\theta_{i k}\right\}$ is a real sequence such that $\theta_{i k}+1>0$, $\prod_{0<t_{k}<t}\left(1+\theta_{i k}\right)$ is an almost periodic continuous function.

The outline of the paper is as follows. In the following section, some definitions and some useful lemmas are listed. In the third section, we first introduce a transformation, where some adjustable real parameters $\rho_{i}>0$ are introduced. After that, by using contraction mapping principle, we derive some sufficient conditions which ensure the existence and uniqueness of positive periodic solution (positive almost periodic solution) of system (1.5) and (1.6). In the fourth section, we derive a set of easily verifiable criteria for the global attractivity of the positive periodic solution (almost periodic solution) of (1.5) and (1.6) by constructing a suitable Lyapunov functional. Finally, we give an example to show our results. Here, We must point out, the idea of introducing parameters is stimulated by the recent works of $[1,26,27]$. However, to the best of the authors knowledge, this is the first time such a technique is applied to the impulsive neutral delays ecosystem.

\section{Preliminaries}

In order to obtain the existence and uniqueness of a periodic solution for system (1.1) and (1.2), we first give some definitions and lemmas:

Definition 2.1 ([21]) A function $N_{i}: R \rightarrow(0,+\infty)$ is said to be a positive solution of (1.5) and (1.6), if the following conditions are satisfied:

1) $N_{i}(t)$ is absolutely continuous on each $\left(t_{k}, t_{k+1}\right)$;

2) for each $k \in Z_{+}, \quad N_{i}\left(t_{k}^{+}\right)$and $N_{i}\left(t_{k}^{-}\right)$exist and $N_{i}\left(t_{k}^{-}\right)=N_{i}\left(t_{k}\right)$

3) $N_{i}(t)$ satisfies the first equation of (1.1) and (1.2) for almost everywhere (for short a.e.) in $[0, \infty] \backslash\left\{t_{k}\right\}$ and satisfies $N_{i}\left(t_{k}^{+}\right)=\left(1+\theta_{i k}\right) N_{i}\left(t_{k}\right)$ for $t=t_{k}$, $k \in Z_{+}=\{1,2, \cdots\}$.

Definition 2.2 Let $N^{*}(t)=\left(N_{1}^{*}(t), \cdots, N_{n}^{*}(t)\right)^{\mathrm{T}}$ be a strictly positive periodic solution (almost periodic solution) of (1.5) and (1.6). We say $N^{*}(t)$ is globally attractive if any other solution $N(t)=\left(N_{1}(t), \cdots, N_{n}(t)\right)^{\mathrm{T}}$ of (1.5) and (1.6) has the property:

$$
\lim _{t \rightarrow+\infty}\left|N_{i}^{*}(t)-N_{i}(t)\right|=0, i=1,2, \cdots, n .
$$

We can easily get the following Lemma 2.1.

Lemma 2.1 The region

$R_{+}^{n}=\left\{N_{i}(t): N_{i}(0)>0, i=1,2, \cdots, n\right\}$ is the positive invariable region of the system (1.5).

Proof. In view of biological population,we obtain $N_{i}(0)>0$. By the system (1.5), we have

$$
\begin{aligned}
N_{i}(t)= & N_{i}(0) \exp \left\{\int _ { 0 } ^ { t } \left[a_{i}(\eta)-b_{i}(\eta) \ln N_{i}(\eta)-\sum_{j=1}^{n} c_{i j}(\eta) \ln N_{j}(\eta)-\sum_{j=1}^{n} d_{i j}(\eta) \ln N_{j}\left(\eta-\gamma_{i j}(\eta)\right)\right.\right. \\
& \left.\left.-\sum_{j=1}^{n} e_{i j}(\eta) \int_{-\infty}^{t} K_{i j}(\eta-s) \ln N_{j}(s) \mathrm{d} s-\sum_{j=1}^{n} f_{i j}(\eta) \frac{\mathrm{d} \ln N_{j}\left(\eta-\delta_{i j}(\eta)\right)}{\mathrm{d} \eta}\right] \mathrm{d} \eta\right\}, t \in\left[0, t_{1}\right], i=1,2, \cdots, n,
\end{aligned}
$$

and

$$
\begin{aligned}
N_{i}(t)= & N_{i}\left(t_{k}\right) \exp \left\{\int _ { t _ { k } } ^ { t } \left[a_{i}(\eta)-b_{i}(\eta) \ln N_{i}(\eta)-\sum_{j=1}^{n} c_{i j}(\eta) \ln N_{j}(\eta)-\sum_{j=1}^{n} d_{i j}(\eta) \ln N_{j}\left(\eta-\gamma_{i j}(\eta)\right)\right.\right. \\
& \left.\left.-\sum_{j=1}^{n} e_{i j}(\eta) \int_{-\infty}^{t} K_{i j}(\eta-s) \ln N_{j}(s) \mathrm{d} s-\sum_{j=1}^{n} f_{i j}(\eta) \frac{\mathrm{d} \ln N_{j}\left(\eta-\delta_{i j}(\eta)\right)}{\mathrm{d} \eta}\right] \mathrm{d} \eta\right\}, t \in\left(t_{k}, t_{k+1}\right], i=1,2, \cdots, n
\end{aligned}
$$




$$
N_{i}\left(t_{k}^{+}\right)=\left(1+\theta_{i k}\right) N_{i}\left(t_{k}\right)>0, k \in N, i=1,2, \cdots, n .
$$

Then the solution of (1.5) is positive.

Under the above hypotheses $\left(\mathrm{H}_{1}\right)-\left(\mathrm{H}_{3}\right)$, I consider the neutral non-impulsive system

$$
\begin{aligned}
\frac{\mathrm{d} y_{i}}{\mathrm{~d} t}= & y_{i}(t)\left[a_{i}(t)-B_{i}(t) \ln y_{i}(t)-\sum_{j=1}^{n} C_{i j}(t) \ln y_{j}(t)-\sum_{j=1}^{n} D_{i j}(t) \ln y_{j}\left(t-\gamma_{i j}(t)\right)\right. \\
& \left.-\sum_{j=1}^{n} E_{i j}(t) \int_{-\infty}^{t} K_{i j}(t-s) \ln y_{j}(s) \mathrm{d} s-\sum_{j=1}^{n} F_{i j}(t) \frac{\mathrm{d} \ln y_{j}\left(t-\delta_{i j}(t)\right)}{\mathrm{d} t}\right],
\end{aligned}
$$

with initial conditions:

$$
\begin{aligned}
& y_{i}(\xi)=\varphi_{i}(\xi), y_{i}^{\prime}(\xi)=\varphi_{i}^{\prime}(\xi), \xi \in[-\tau, 0], \varphi_{i}(0)>0, \\
& \varphi_{i} \in C\left([-\tau, 0), R^{+}\right) \cap C^{1}\left([-\tau, 0), R^{+}\right), i=1,2,3, \cdots, n,
\end{aligned}
$$

where

$$
\begin{aligned}
& B_{i}(t)=b_{i}(t) \prod_{0<t_{k}<t}\left(1+\theta_{i k}\right), C_{i j}(t)=c_{i j}(t) \prod_{0<t_{k}<t}\left(1+\theta_{i k}\right), D_{i j}(t)=d_{i j}(t) \prod_{0<t_{k}<t}\left(1+\theta_{i k}\right), \\
& E_{i j}(t)=e_{i j}(t) \prod_{0<t_{k}<t-\gamma_{i j}(t)}\left(1+\theta_{i k}\right), F_{i j}(t)=f_{i j}(t) \prod_{0<t_{k}<t-\delta_{i j}(t)}\left(1+\theta_{i k}\right) .
\end{aligned}
$$

By a solution $y_{i}(t), i=1,2, \cdots, n$, of (2.1) and (2.2), it means an absolutely continuous function $y_{i}(t)$, $i=1,2, \cdots, n$, defined on $[-\tau, 0]$ that satisfies $(2.1)$ a.e., for $t \geq 0$, and $y_{i}(\xi)=\varphi_{i}(\xi), y_{i}^{\prime}(\xi)=\varphi_{i}^{\prime}(\xi)$ on $[-\tau, 0]$.

The following lemmas will be used in the proofs of our results, The proof of the first lemma is similar to that of Theorem 1 in [20].

Lemma 2.2 Suppose that $\left(\mathrm{H}_{1}\right)-\left(\mathrm{H}_{3}\right)$ hold. Then

1) if $y_{i}(t)(i=1,2, \cdots, n)$ is a solution of (2.1) and (2.2) on $[-\tau,+\infty)$, then

$$
N_{i}(t)=\prod_{0<t_{k}<t}\left(1+\theta_{i k}\right) y_{i}(t)(i=1,2, \cdots, n)
$$

is a solution of $(1.5)$ and (1.6) on $[-\tau,+\infty)$.

2) if $N_{i}(t)(i=1,2, \cdots, n)$ is a solution of (1.5) and (1.6) on $[-\tau,+\infty)$, then

$$
y_{i}(t)=\prod_{0<t_{k}<t}\left(1+\theta_{i k}\right) N_{i}(t)(i=1,2, \cdots, n)
$$

is a solution of (2.1) and (2.2) on $[-\tau,+\infty)$.

Proof. Its proof is similar to that of Theorem 1 in [20], here we omit it.

Lemma 2.3 ([28]) Suppose $\sigma \in C_{\omega}^{1}$ and $\sigma^{\prime}(t)<1$, $t \in[0, \omega]$. Then the function $t-\sigma(t)$ has a unique inverse $\mu(t)$ satisfying $\mu \in C(R, R)$ with $\mu(a+\omega)=\mu(a)+\omega \quad \forall a \in R$.

Proof. Its proof is similar to that of Lemma 2.4 in [29], here we omit it.

Lemma 2.4 (Barbalat's Lemma [30]) Let $f(t)$ be a nonnegative function defined on $[0,+\infty)$ such that $f(t)$ is integrable and uniformly continuous on $[0,+\infty)$, then $\lim _{t \rightarrow+\infty} f(t)=0$.
Lemma 2.5 Assume that $u(t), \tau(t)$ are all continuously differentiable $\omega$-periodic functions, $a(t)$, $b(t)$ are both nonnegative continuous $\omega$-periodic functions such that $\int_{0}^{\omega} a(t) \mathrm{d} t>0$, then

$$
\begin{aligned}
& \int_{-\infty}^{t} \mathrm{e}^{-\int_{s}^{t} a(\xi) \mathrm{d} \xi} b(s) u^{\prime}(s-\tau(s)) \mathrm{d} s \\
& =c(t) u(t-\tau(t)) \\
& \quad-\int_{-\infty}^{t} \mathrm{e}^{-\int_{s}^{t} a(\xi) \mathrm{d} \xi}\left[a(s) c(s)+c^{\prime}(s)\right] u(s-\tau(s)) \mathrm{d} s,
\end{aligned}
$$

where $c(t)=\frac{b(t)}{1-\tau^{\prime}(t)}$.

Proof. As

$$
\begin{aligned}
& \int_{-\infty}^{t} \mathrm{e}^{-\int_{s}^{t} a(\xi) \mathrm{d} \xi} b(s) u^{\prime}(s-\tau(s)) \mathrm{d} s \\
& =\int_{-\infty}^{t} \mathrm{e}^{-\int_{s}^{t} a(\xi) \mathrm{d} \xi} c(s) \mathrm{d} u(s-\tau(s)) \\
& =\left.\mathrm{e}^{-\int_{s}^{t} a(\xi) \mathrm{d} \xi} c(s) u(s-\tau(s))\right|_{-\infty} ^{t} \\
& \quad-\int_{-\infty}^{t} u(s-\tau(s)) \mathrm{d}\left(\mathrm{e}^{-\int_{s}^{t} a(\xi) \mathrm{d} \xi} c(s)\right) \\
& =\left.\mathrm{e}^{-\int_{s}^{t} a(\xi) \mathrm{d} \xi} c(s) u(s-\tau(s))\right|_{-\infty} ^{t} \\
& \quad-\int_{-\infty}^{t} u(s-\tau(s))\left[a(s) c(s)+c^{\prime}(s)\right] \mathrm{e}^{-\int_{s}^{t} a(\xi) \mathrm{d} \xi} \mathrm{d} s,
\end{aligned}
$$

Denote $m=\mathrm{e}^{-\int_{0}^{\omega} a(t) \mathrm{d} t}$, then from $a(t) \geq 0$,

$\int_{0}^{\omega} a(t) \mathrm{d} t>0$, it follows $m<1$. Also, when $t \geq s$ without loss of generality, we may assume $s+n \omega \leq t \leq s+(n+1) \omega$, thus 


$$
\begin{aligned}
& \left|\mathrm{e}^{-\int_{s}^{t} a(\xi) \mathrm{d} \xi} c(s) u(s-\tau(s))\right| \leq \mathrm{e}^{-\int_{s}^{t} a(\xi) \mathrm{d} \xi}\|c\|\|u\| \\
& =\mathrm{e}^{-\sum_{j=1}^{n-1} \int_{s+j \omega}^{s+(j+1) \omega} a(\xi) \mathrm{d} \xi-\int_{s+n \omega}^{t} a(\xi) \mathrm{d} \xi}\|c\|\|u\| \\
& =k^{n} \mathrm{e}^{-\int_{s+n}^{t} a(\xi) \mathrm{d} \xi}\|c\|\|u\| \leq k^{n}\|c\|\|u\| .
\end{aligned}
$$

Therefore

$$
\lim _{s \rightarrow-\infty} \mathrm{e}^{-\int_{s}^{t} a(\xi) \mathrm{d} \xi} c(s) u(s-\tau(s))=0,
$$

and so, from (2.9) it follows:

$$
\begin{aligned}
& \int_{-\infty}^{t} \mathrm{e}^{-\int_{s}^{t} a(\xi) \mathrm{d} \xi} b(s) u^{\prime}(s-\tau(s)) \mathrm{d} s \\
& =c(t) u(t-\tau(t)) \\
& \quad-\int_{-\infty}^{t} \mathrm{e}^{-\int_{s}^{t} a(\xi) \mathrm{d} \xi}\left[a(s) c(s)+c^{\prime}(s)\right] u(s-\tau(s)) \mathrm{d} s .
\end{aligned}
$$

The proof is complete.

Lemma 2.6 Assume that $u(t)$ are all continuously differentiable almost periodic functions, $a(t), b(t)$ are both nonnegative continuous almost periodic functions such that $m(a(t))>0, \tau$ is positive number, then

$$
\begin{aligned}
& \int_{-\infty}^{t} \mathrm{e}^{-\int_{s}^{t} a(\xi) \mathrm{d} \xi} b(s) u^{\prime}(s-\tau(s)) \mathrm{d} s \\
& =c(t) u(t-\tau(t)) \\
& \quad-\int_{-\infty}^{t} \mathrm{e}^{-\int_{s}^{t} a(\xi) \mathrm{d} \xi}\left[a(s) b(s)+b^{\prime}(s)\right] u(s-\tau(s)) \mathrm{d} s,
\end{aligned}
$$

where $m(a(t))=\lim _{T \rightarrow+\infty} \frac{1}{T} \int_{t}^{t+T} a(\tau) \mathrm{d} \tau$.

Proof. Similar to the proof of Lemma 2.5, we omit it here.

\section{Main Theorem}

Here, we take the transformation $y_{i}(t)=\exp \left\{\rho_{i} x_{i}(t)\right\}$, then (2.1) can be rewritten in the following form

$$
\begin{aligned}
& \frac{\mathrm{d} x_{i}(t)}{\mathrm{d} t}=-\left[b_{i}(t)+C_{i j}(t)\right] x_{i}(t)-\sum_{j=1, j \neq i}^{n} \frac{\rho_{j}}{\rho_{i}} C_{i j}(t) x_{j}(t) \\
&-\sum_{j=1}^{n} \frac{\rho_{j}}{\rho_{i}} D_{i j}(t) x_{j}\left(t-\gamma_{i j}(t)\right) \\
&-\sum_{j=1}^{n} \frac{\rho_{j}}{\rho_{i}} E_{i j}(t) \int_{-\infty}^{t} K_{i j}(t-s) x_{j}(s) \mathrm{d} s \\
&-\sum_{j=1}^{n} \frac{\rho_{j}}{\rho_{i}} F_{i j}(t)\left(1-\delta_{i j}^{\prime}(t)\right) x_{j}^{\prime}\left(t-\delta_{i j}(t)\right)+\frac{a_{i}(t)}{\rho_{i}}, \\
& i=1,2, \cdots, n .
\end{aligned}
$$

Obviously, the existence, uniqueness and global attractivity of positive periodic solution (almost periodic solution) of system (1.5) is equivalent to the existence, uniqueness and global attractivity of periodic solution (almost periodic solution) of system (3.1).

For $u(t)=\left(u_{1}(t), u_{2}(t), \cdots, u_{n}(t)\right)^{\mathrm{T}} \in C_{\omega}^{1}$, let us consider the equation

$$
\begin{aligned}
& \frac{\mathrm{d} x_{i}(t)}{\mathrm{d} t}=-\left[b_{i}(t)+C_{i i}(t)\right] x_{i}(t)-\sum_{j=1, j \neq i}^{n} \frac{\rho_{j}}{\rho_{i}} C_{i j}(t) u_{j}(t) \\
&-\sum_{j=1}^{n} \frac{\rho_{j}}{\rho_{i}} D_{i j}(t) u_{j}\left(t-\gamma_{i j}(t)\right) \\
&-\sum_{j=1}^{n} \frac{\rho_{j}}{\rho_{i}} E_{i j}(t) \int_{-\infty}^{t} K_{i j}(t-s) u_{j}(s) \mathrm{d} s \\
&-\sum_{j=1}^{n} \frac{\rho_{j}}{\rho_{i}} F_{i j}(t)\left(1-\delta_{i j}^{\prime}(t)\right) u_{j}^{\prime}\left(t-\delta_{i j}(t)\right)+\frac{a_{i}(t)}{\rho_{i}}, \\
& i=1,2, \cdots, n .
\end{aligned}
$$

Since $b_{i}(t)+C_{i i}(t)>0, \int_{0}^{\omega}\left[b_{i}(t)+C_{i i}(t)\right] \mathrm{d} t>0$, it follows that the linear system of system (3.2)

$$
\frac{\mathrm{d} x_{i}}{\mathrm{~d} t}=-\left[b_{i}(t)+C_{i i}(t)\right] x_{i}(t), i=1,2, \cdots, n,
$$

admits exponential dichotomies on R, and so, system (3.3) has a unique continuous periodic solution $x_{i u}(t)$, which can be expressed as

$$
\begin{gathered}
x_{i u}(t)=\int_{-\infty}^{t} \mathrm{e}^{-\int_{\tau}^{t}\left[b_{i}(\xi)+C_{i i}(\xi)\right] \mathrm{d} \xi} f_{i u}(\tau) \mathrm{d} \tau, \\
i=1,2, \cdots, n,
\end{gathered}
$$

where

$$
\begin{aligned}
f_{i u}(\tau)= & -\sum_{j=1, j \neq i}^{n} \frac{\rho_{j}}{\rho_{i}} C_{i j}(\tau) u_{j}(\tau) \\
& -\sum_{j=1}^{n} \frac{\rho_{j}}{\rho_{i}} D_{i j}(\tau) u_{j}\left(\tau-\gamma_{i j}(\tau)\right) \\
& -\sum_{j=1}^{n} \frac{\rho_{j}}{\rho_{i}} E_{i j}(\tau) \int_{-\infty}^{\tau} K_{i j}(\tau-s) u_{j}(s) \mathrm{d} s \\
& -\sum_{j=1}^{n} \frac{\rho_{j}}{\rho_{i}} F_{i j}(\tau)\left(1-\delta_{i j}^{\prime}(\tau)\right) u_{j}^{\prime}\left(\tau-\delta_{i j}(\tau)\right)+\frac{a_{i}(\tau)}{\rho_{i}}, \\
i=1,2, \cdots, n . &
\end{aligned}
$$

Now, by using Lemma 2.5, $x_{i u}(t)$ can also be expressed as

$$
\begin{aligned}
& x_{i u}(t)=-\sum_{j=1}^{n} \frac{\rho_{j}}{\rho_{i}} F_{i j}(t) u_{j}\left(t-\delta_{i j}(t)\right) \\
&+\int_{-\infty}^{t} \mathrm{e}^{-\int_{\tau}^{t}\left[b_{i}(\xi)+C_{i i}(\xi)\right] \mathrm{d} \xi} g_{i u}(\tau) \mathrm{d} \tau, \\
& i=1,2, \cdots, n,
\end{aligned}
$$

where 


$$
\begin{aligned}
g_{i u}(\tau)= & -\sum_{j=1, j \neq i}^{n} \frac{\rho_{j}}{\rho_{i}} C_{i j}(\tau) u_{j}(\tau)-\sum_{j=1}^{n} \frac{\rho_{j}}{\rho_{i}} D_{i j}(\tau) u_{j}\left(\tau-\gamma_{i j}(\tau)\right)-\sum_{j=1}^{n} \frac{\rho_{j}}{\rho_{i}} E_{i j}(\tau) \int_{-\infty}^{t} K_{i j}(\tau-s) u_{j}(s) \mathrm{d} s \\
& +\sum_{j=1}^{n} \frac{\rho_{j}}{\rho_{i}}\left\{F_{i j}(\tau)\left[b_{i}(\tau)+C_{i j}(\tau)\right]+F_{i j}^{\prime}(\tau)\right\} u_{j}\left(\tau-\delta_{i j}(\tau)\right)+\frac{a_{i}(t)}{\rho_{i}}, \quad i=1,2, \cdots, n .
\end{aligned}
$$

Our main result on the global existence of a positive periodic solution of (1.5) and (1.6) is stated as follows.

Theorem 3.1 In addition to $\left(\mathrm{H}_{1}\right)-\left(\mathrm{H}_{3}\right)$, assume further that there exist positive constants $\rho_{i}(i=1,2, \cdots, n)$, such that

$\left(\mathrm{H}_{7}\right) \max _{t \in R} \int_{-\infty}^{t} \mathrm{e}^{-\int_{\tau}^{t}\left[b_{i}(\xi)+C_{i i}(\xi)\right] \mathrm{d} \xi} q_{i}(\tau) \mathrm{d} \tau<1-\left\|\sum_{j=1}^{n} \frac{\rho_{j}}{\rho_{i}} F_{i j}(t)\right\|_{1}$.

Then (1.5) has a unique positive $\omega$-periodic solution with strictly positive components, say

$$
N^{*}(t)=\left(N_{1}^{*}(t), N_{2}^{*}(t), \cdots, N_{n}^{*}(t)\right)^{\mathrm{T}},
$$

where

$$
q_{i}(\tau)=\sum_{j=1, j \neq i}^{n} \frac{\rho_{j}}{\rho_{i}} C_{i j}(\tau)+\sum_{j=1}^{n} \frac{\rho_{j}}{\rho_{i}}\left\{D_{i j}(\tau)+E_{i j}(\tau)+F_{i j}(\tau)\left[b_{i}(\tau)+C_{i i}(\tau)\right]+\left|F_{i j}^{\prime}(\tau)\right|\right\},
$$

and

$$
\left.\left\|\sum_{j=1}^{n} \frac{\rho_{j}}{\rho_{i}} F_{i j}(t)\right\|_{1}=\max _{t \in[0, \omega]}\left\{\mid \sum_{j=1}^{n} \frac{\rho_{j}}{\rho_{i}} F_{i j}(t)\right\}\right\} .
$$

Proof. For $u(t)=\left(u_{1}(t), u_{2}(t), \cdots, u_{n}(t)\right)^{\mathrm{T}} \in C_{\omega}$, from (3.6), we know that

$$
\begin{gathered}
x_{i u}(t)=-\sum_{j=1}^{n} \frac{\rho_{j}}{\rho_{i}} F_{i j}(t) u_{j}\left(t-\delta_{i j}(t)\right)+\int_{-\infty}^{t} \mathrm{e}^{-\int_{\tau}^{t}\left[b_{i}(\xi)+C_{i i}(\xi)\right] \mathrm{d} \xi} g_{i u}(\tau) \mathrm{d} \tau, \\
i=1,2, \cdots, n,
\end{gathered}
$$

where $g_{i u}(\tau)$ are defined by (3.7), is a continuous $\omega$ periodic function, and so $x_{u}(t)=\left(x_{1 u}(t), x_{2 u}(t), \cdots, x_{n u}(t)\right)^{\mathrm{T}} \in C_{\omega}$. Now define the mapping $\psi: C_{\omega} \rightarrow C_{\omega}$ as follows:

$$
\begin{aligned}
\psi u(t)= & x_{u}(t), u(t) \in C_{\omega} . \quad(3.9) \quad \text { the conditions of Theorem 3.1 it follows: } \\
\left\|\psi u-\psi u^{*}\right\|_{1}= & \max _{t \in[0, \omega]}\left\{\left|\sum_{j=1}^{n} \frac{\rho_{j}}{\rho_{1}} F_{1 j}(t)\left[u_{j}\left(t-\delta_{1 j}(t)\right)-u_{j}^{*}\left(t-\delta_{1 j}(t)\right)\right]\right|\right. \\
& +\int_{-\infty}^{t} \mathrm{e}^{-\int_{\tau}^{t}\left[b_{1}(\xi)+C_{11}(\xi)\right] \mathrm{d} \xi}\left|g_{1 u}(\tau)-g_{1 u^{*}}(\tau)\right| \mathrm{d} \tau, \cdots,\left|\sum_{j=1}^{n} \frac{\rho_{j}}{\rho_{n}} F_{n j}(t)\left[u_{j}\left(t-\delta_{n j}(t)\right)-u_{j}^{*}\left(t-\delta_{n j}(t)\right)\right]\right| \\
& \left.\left.+\int_{-\infty}^{t} \mathrm{e}^{-\int_{\tau}^{t}\left[b_{n}(\xi)+C_{n n}(\xi)\right] \mathrm{d} \xi}\left|g_{n u}(\tau)-g_{n u^{*}}(\tau)\right| \mathrm{d} \tau\right\}\right) \\
\leq & \max _{t \in[0, \omega]}\left\{\left[\left\|\sum_{j=1}^{n} \frac{\rho_{j}}{\rho_{1}} F_{1 j}(t)\right\|+\int_{-\infty}^{t} \mathrm{e}^{-\int_{\tau}^{t} \tau\left[b_{1}(\xi)+C_{11}(\xi)\right] \mathrm{d} \xi} q(\tau) \mathrm{d} \tau\right]\left\|u-u^{*}\right\|_{1},\right. \\
& {\left.\left[\left\|\sum_{j}^{n} \frac{\rho_{j}}{\rho_{n}} F_{n j}(t)\right\|_{1}+\int_{-\infty}^{t} \mathrm{e}^{-\int_{\tau}^{t}\left[b_{n}(\xi)+C_{n n}(\xi)\right] \mathrm{d} \xi} q(\tau) \mathrm{d} \tau\right]\left\|u-u^{*}\right\|_{1}\right\} } \\
< & \left\|u-u^{*}\right\|_{1},
\end{aligned}
$$

Following we will prove the mapping $\psi$ is a contraction mapping. In fact, for any $u(t)=\left(u_{1}(t), u_{2}(t), \cdots, u_{n}(t)\right)^{\mathrm{T}}$ and $u^{*}(t)=\left(u_{1}^{*}(t), u_{2}^{*}(t), \cdots, u_{n}^{*}(t)\right)^{\mathrm{T}}$ from (3.8), (3.9) and

where 


$$
\begin{aligned}
\left\|g_{i u}(\tau)-g_{i u^{*}}(\tau)\right\|_{1} \leq & \sum_{j=1, j \neq i}^{n} \frac{\rho_{j}}{\rho_{i}} C_{i j}(\tau)\left|u_{j}(\tau)-u_{j}^{*}(\tau)\right|+\sum_{j=1}^{n} \frac{\rho_{j}}{\rho_{i}} D_{i j}(\tau)\left|u_{j}\left(\tau-\gamma_{i j}(\tau)\right)-u_{j}^{*}\left(\tau-\gamma_{i j}(\tau)\right)\right| \\
& +\sum_{j=1}^{n} \frac{\rho_{j}}{\rho_{i}} E_{i j}(\tau) \int_{-\infty}^{t} K_{i j}(\tau-s)\left|u_{j}(s)+u_{j}^{*}(s)\right| \mathrm{d} s \\
& +\sum_{j=1}^{n} \frac{\rho_{j}}{\rho_{i}}\left\{F_{i j}(\tau)\left[b_{i}(\tau)+C_{i j}(\tau)\right]+F_{i j}^{\prime}(\tau)\right\}\left|u_{j}\left(\tau-\delta_{i j}(\tau)\right)-u_{j}^{*}\left(\tau-\delta_{i j}(\tau)\right)\right| \\
\leq & \sum_{j=1, j \neq i}^{n} \frac{\rho_{j}}{\rho_{i}} C_{i j}(\tau)\left\|u_{j}-u_{j}^{*}\right\|_{1}+\sum_{j=1}^{n} \frac{\rho_{j}}{\rho_{i}}\left\{D_{i j}(\tau)+E_{i j}(\tau)+F_{i j}(\tau)\left[b_{i}(\tau)+C_{i i}(\tau)\right]+\left|F_{i j}^{\prime}(\tau)\right|\right\}\left\|u_{j}-u_{j}^{*}\right\|_{1} \\
= & q_{i}(\tau)\left\|u_{j}-u_{j}^{*}\right\|_{1}, \quad i=1,2, \cdots, n .
\end{aligned}
$$

That is

$$
\left\|\psi u-\psi u^{*}\right\|_{1}<\left\|u-u^{*}\right\|_{1}
$$

This shows that $\psi$ is a contraction mapping. Hence, there exists a unique fixed point $x(t)=\left(x_{1}(t), \cdots, x_{n}(t)\right)^{\mathrm{T}} \in C_{\omega}$ such that $\psi x=x$, that is

$$
\begin{aligned}
& x_{i}(t)=-\sum_{j=1}^{n} \frac{\rho_{j}}{\rho_{i}} F_{i j}(t) u_{j}\left(t-\delta_{i j}(t)\right)+\int_{-\infty}^{t} \mathrm{e}^{-\int_{\tau}^{t}\left[b_{i}(\xi)+C_{i i}(\xi)\right] \mathrm{d} \xi}\left[-\sum_{j=1, j \neq i}^{n} \frac{\rho_{j}}{\rho_{i}} C_{i j}(\tau) u_{j}(\tau)\right. \\
&-\sum_{j=1}^{n} \frac{\rho_{j}}{\rho_{i}} D_{i j}(\tau) u_{j}\left(\tau-\gamma_{i j}(\tau)\right)-\sum_{j=1}^{n} \frac{\rho_{j}}{\rho_{i}} E_{i j}(\tau) \int_{-\infty}^{t} K_{i j}(\tau-s) u_{j}(s) \mathrm{d} s \\
&\left.+\sum_{j=1}^{n} \frac{\rho_{j}}{\rho_{i}}\left\{F_{i j}(\tau)\left[b_{i}(\tau)+C_{i j}(\tau)\right]+F_{i j}^{\prime}(\tau)\right\} u_{j}\left(\tau-\delta_{i j}(\tau)\right)+\frac{a_{i}(t)}{\rho_{i}}\right] \mathrm{d} \tau, \\
& i=1,2, \cdots, n .
\end{aligned}
$$

Following, we prove $x(t)=\left(x_{1}(t), \cdots, x_{n}(t)\right)^{\mathrm{T}} \in C_{\omega}$ is the periodic solution of system (3.1). Noticing that (3.12) is equivalent to

$$
\begin{aligned}
& x_{i}(t)+\sum_{j=1}^{n} \frac{\rho_{j}}{\rho_{i}} F_{i j}(t) u_{j}\left(t-\delta_{i j}(t)\right) \\
& =\int_{-\infty}^{t} \mathrm{e}^{-f_{\tau}^{t}\left[b_{i}(\xi)+C_{i i}(\xi)\right] \mathrm{d} \xi}\left[-\sum_{j=1, j \neq i}^{n} \frac{\rho_{j}}{\rho_{i}} C_{i j}(\tau) u_{j}(\tau)-\sum_{j=1}^{n} \frac{\rho_{j}}{\rho_{i}} D_{i j}(\tau) u_{j}\left(\tau-\gamma_{i j}(\tau)\right)\right. \\
& \quad-\sum_{j=1}^{n} \frac{\rho_{j}}{\rho_{i}} E_{i j}(\tau) \int_{-\infty}^{t} K_{i j}(\tau-s) u_{j}(s) \mathrm{d} s \\
& \left.\quad+\sum_{j=1}^{n} \frac{\rho_{j}}{\rho_{i}}\left\{F_{i j}(\tau)\left[b_{i}(\tau)+C_{i j}(\tau)\right]+F_{i j}^{\prime}(\tau)\right\} u_{j}\left(\tau-\delta_{i j}(\tau)\right)+\frac{a_{i}(t)}{\rho_{i}}\right] \mathrm{d} \tau, \\
& i=1,2, \cdots, n .
\end{aligned}
$$

From the right-hand sides of (3.13), we know that

$$
x_{i}(t)+\sum_{j=1}^{n} \frac{\rho_{j}}{\rho_{i}} F_{i j}(t) u_{j}\left(t-\delta_{i j}(t)\right)
$$

is differentiable. And so, from (3.13) it follows that 


$$
\begin{aligned}
& \frac{\mathrm{d} x_{i}(t)}{\mathrm{d} t}+\sum_{j=1}^{n} \frac{\rho_{j}}{\rho_{i}} F_{i j}^{\prime}(t) u_{j}\left(t-\delta_{i j}(t)\right)+\sum_{j=1}^{n} \frac{\rho_{j}}{\rho_{i}} F_{i j}(t)\left(1-\delta_{i j}^{\prime}(t)\right) u_{j}\left(t-\delta_{i j}(t)\right) \\
& =\frac{\mathrm{d}}{\mathrm{d} t}\left[x_{i}(t)+\sum_{j=1}^{n} \frac{\rho_{j}}{\rho_{i}} F_{i j}(t) u_{j}\left(t-\delta_{i j}(t)\right)\right] \\
& =-\sum_{j=1, j \neq i}^{n} \frac{\rho_{j}}{\rho_{i}} C_{i j}(t) u_{j}(t)-\sum_{j=1}^{n} \frac{\rho_{j}}{\rho_{i}} D_{i j}(t) u_{j}\left(t-\gamma_{i j}(t)\right)-\sum_{j=1}^{n} \frac{\rho_{j}}{\rho_{i}} E_{i j}(t) \int_{-\infty}^{t} K_{i j}(t-s) u_{j}(s) \mathrm{d} s \\
& +\sum_{j=1}^{n} \frac{\rho_{j}}{\rho_{i}}\left\{F_{i j}(t)\left[b_{i}(t)+C_{i j}(t)\right]+F_{i j}^{\prime}(t)\right\} u_{j}\left(t-\delta_{i j}(t)\right)+\frac{a_{i}(t)}{\rho_{i}} \\
& -\left[b_{i}(t)+C_{i i}(t)\right] \int_{-\infty}^{t} \mathrm{e}^{-\int_{\tau}^{t}\left[b_{i}(\xi)+C_{i i}(\xi)\right] \mathrm{d} \xi}\left[-\sum_{j=1, j \neq i}^{n} \frac{\rho_{j}}{\rho_{i}} C_{i j}(\tau) u_{j}(\tau)-\sum_{j=1}^{n} \frac{\rho_{j}}{\rho_{i}} D_{i j}(\tau) u_{j}\left(\tau-\gamma_{i j}(\tau)\right)\right. \\
& \left.-\sum_{j=1}^{n} \frac{\rho_{j}}{\rho_{i}} E_{i j}(\tau) \int_{-\infty}^{t} K_{i j}(\tau-s) u_{j}(s) \mathrm{d} s+\sum_{j=1}^{n} \frac{\rho_{j}}{\rho_{i}}\left\{F_{i j}(\tau)\left[b_{i}(\tau)+C_{i j}(\tau)\right]+F_{i j}^{\prime}(\tau)\right\} u_{j}\left(\tau-\delta_{i j}(\tau)\right)+\frac{a_{i}(t)}{\rho_{i}}\right] \mathrm{d} \tau \\
& =-\sum_{j=1, j \neq i}^{n} \frac{\rho_{j}}{\rho_{i}} C_{i j}(t) u_{j}(t)-\sum_{j=1}^{n} \frac{\rho_{j}}{\rho_{i}} D_{i j}(t) u_{j}\left(t-\gamma_{i j}(t)\right)-\sum_{j=1}^{n} \frac{\rho_{j}}{\rho_{i}} E_{i j}(t) \int_{-\infty}^{t} K_{i j}(t-s) u_{j}(s) \mathrm{d} s \\
& +\sum_{j=1}^{n} \frac{\rho_{j}}{\rho_{i}}\left\{F_{i j}(t)\left[b_{i}(t)+C_{i j}(t)\right]+F_{i j}^{\prime}(t)\right\} u_{j}\left(t-\delta_{i j}(t)\right)+\frac{a_{i}(t)}{\rho_{i}} \\
& -\left[b_{i}(t)+C_{i i}(t)\right]\left[x_{i}(t)+\sum_{j=1}^{n} \frac{\rho_{j}}{\rho_{i}} F_{i j}(t) u_{j}\left(t-\delta_{i j}(t)\right)\right], i=1,2, \cdots, n,
\end{aligned}
$$

here using the equality (3.13) again. That is

$$
\begin{aligned}
\frac{\mathrm{d} x_{i}(t)}{\mathrm{d} t}= & -\left[b_{i}(t)+C_{i i}(t)\right] x_{i}(t)-\sum_{j=1, j \neq i}^{n} \frac{\rho_{j}}{\rho_{i}} C_{i j}(t) u_{j}(t)-\sum_{j=1}^{n} \frac{\rho_{j}}{\rho_{i}} D_{i j}(t) u_{j}\left(t-\gamma_{i j}(t)\right) \\
& -\sum_{j=1}^{n} \frac{\rho_{j}}{\rho_{i}} E_{i j}(t) \int_{-\infty}^{t} K_{i j}(t-s) u_{j}(s) \mathrm{d} s-\sum_{j=1}^{n} \frac{\rho_{j}}{\rho_{i}} F_{i j}(t)\left(1-\delta_{i j}^{\prime}(t)\right) u_{j}^{\prime}\left(t-\delta_{i j}(t)\right)+\frac{a_{i}(t)}{\rho_{i}}, i=1,2, \cdots, n .
\end{aligned}
$$

This shows that $x(t)=\left(x_{1}(t), \cdots, x_{n}(t)\right)^{\mathrm{T}}$ is continuously differentiable $\omega$-periodic function and satisfies Equation (3.1). Therefore, $x(t)=\left(x_{1}(t), \cdots, x_{n}(t)\right)^{\mathrm{T}}$ is the unique continuously differentiable $\omega$-periodic solution of system (3.1), and so,

$$
y(t)=\left(\exp \left\{\rho_{1} x_{1}(t)\right\}, \cdots, \exp \left\{\rho_{n} x_{n}(t)\right\}\right)^{\mathrm{T}}
$$

is the unique positive $\omega$-periodic solution of system (2.1), from Lemma 2.2,

$$
\begin{aligned}
N(t)= & \left(\prod_{0<t_{k}<t}\left(1+\theta_{i k}\right) \exp \left\{\rho_{1} x_{1}(t)\right\}, \cdots,\right. \\
& \left.\prod_{0<t_{k}<t}\left(1+\theta_{i k}\right) \exp \left\{\rho_{n} x_{n}(t)\right\}\right)^{\mathrm{T}}
\end{aligned}
$$

is the unique positive $\omega$-periodic solution of system (1.5). The proof is complete.

As a direct corollary of Theorem 3.1, one has

Corollary 3.1 In addition to $\left(\mathrm{H}_{1}\right)-\left(\mathrm{H}_{3}\right)$, assume further that there exist positive constants $\rho_{i}(i=1,2, \cdots, n)$, such that

$$
\begin{aligned}
q_{i}(t) & =\sum_{j=1, j \neq i}^{n} \frac{\rho_{j}}{\rho_{i}} C_{i j}(t)+\sum_{j=1}^{n} \frac{\rho_{j}}{\rho_{i}}\left\{D_{i j}(t)+E_{i j}(t)+F_{i j}(t)\left[b_{i}(t)+C_{i i}(t)\right]+\left|F_{i j}^{\prime}(t)\right|\right\} \\
& <\left[b_{i}(t)+C_{i i}(t)\right]\left(1-\left\|\sum_{j=1}^{n} \frac{\rho_{j}}{\rho_{i}} F_{i j}(t)\right\|_{1}\right) .
\end{aligned}
$$

Then (1.5) has a unique positive $\omega$-periodic solution with strictly positive components.

Our next theorem concerned with the existence of unique positive almost periodic solution of systems (1.5) and (1.6).

Let $u(t)=\left(u_{1}(t), u_{2}(t), \cdots, u_{n}(t)\right)^{\mathrm{T}}$ be any continuously differentiable almost periodic function, and consider equation, 


$$
\begin{aligned}
\frac{\mathrm{d} x_{i}(t)}{\mathrm{d} t}= & -\left[b_{i}(t)+C_{i i}(t)\right] x_{i}(t)-\sum_{j=1, j \neq i}^{n} \frac{\rho_{j}}{\rho_{i}} C_{i j}(t) u_{j}(t) \\
& -\sum_{j=1}^{n} \frac{\rho_{j}}{\rho_{i}} D_{i j}(t) u_{j}\left(t-\gamma_{i j}(t)\right)-\sum_{j=1}^{n} \frac{\rho_{j}}{\rho_{i}} E_{i j}(t) \int_{-\infty}^{t} K_{i j}(t-s) u_{j}(s) \mathrm{d} s \\
& -\sum_{j=1}^{n} \frac{\rho_{j}}{\rho_{i}} F_{i j}(t)\left(1-\delta_{i j}^{\prime}(t)\right) u_{j}^{\prime}\left(t-\delta_{i j}(t)\right)+\frac{a_{i}(t)}{\rho_{i}}, \quad i=1,2, \cdots, n .
\end{aligned}
$$

Since $m\left(b_{i}(t)+d_{i i}(t)\right)>0$, it follows that the linear system of system (3.15)

$$
\frac{\mathrm{d} x_{i}}{\mathrm{~d} t}=-\left[b_{i}(t)+C_{i i}(t)\right] x_{i}(t), i=1,2, \cdots, n,
$$

admits exponential dichotomies on $\mathrm{R}$, and so, system (3.16) has a unique continuous almost periodic solution $x_{i u}(t)$, which can be expressed as

$$
x_{i u}(t)=\int_{-\infty}^{t} \mathrm{e}^{-\int_{\tau}^{t}\left[b_{i}(\xi)+C_{i i}(\xi)\right] \mathrm{d} \xi} f_{i u}(\tau) \mathrm{d} \tau, \quad i=1,2, \cdots, n,
$$

where

$$
\begin{aligned}
f_{i u}(\tau)= & -\sum_{j=1, j \neq i}^{n} \frac{\rho_{j}}{\rho_{i}} C_{i j}(\tau) u_{j}(\tau)-\sum_{j=1}^{n} \frac{\rho_{j}}{\rho_{i}} D_{i j}(\tau) u_{j}\left(\tau-\gamma_{i j}(\tau)\right)-\sum_{j=1}^{n} \frac{\rho_{j}}{\rho_{i}} E_{i j}(\tau) \int_{-\infty}^{t} K_{i j}(\tau-s) u_{j}(s) \mathrm{d} s \\
& -\sum_{j=1}^{n} \frac{\rho_{j}}{\rho_{i}} F_{i j}(\tau)\left(1-\delta_{i j}^{\prime}(\tau)\right) u_{j}^{\prime}\left(\tau-\delta_{i j}(\tau)\right)+\frac{a_{i}(\tau)}{\rho_{i}}, i=1,2, \cdots, n .
\end{aligned}
$$

Now, by using Lemma $2.5, x_{i u}(t)$ can also be expressed as

$$
x_{i u}(t)=-\sum_{j=1}^{n} \frac{\rho_{j}}{\rho_{i}} F_{i j}(t) u_{j}\left(t-\delta_{i j}(t)\right)+\int_{-\infty}^{t} \mathrm{e}^{-\int_{\tau}^{t}\left[b_{i}(\xi)+C_{i i}(\xi)\right] \mathrm{d} \xi} g_{i u}(\tau) \mathrm{d} \tau, \quad i=1,2, \cdots, n
$$

where

$$
\begin{aligned}
g_{i u}(\tau)= & -\sum_{j=1, j \neq i}^{n} \frac{\rho_{j}}{\rho_{i}} C_{i j}(\tau) u_{j}(\tau)-\sum_{j=1}^{n} \frac{\rho_{j}}{\rho_{i}} D_{i j}(\tau) u_{j}\left(\tau-\gamma_{i j}(\tau)\right)-\sum_{j=1}^{n} \frac{\rho_{j}}{\rho_{i}} E_{i j}(\tau) \int_{-\infty}^{t} K_{i j}(\tau-s) u_{j}(s) \mathrm{d} s \\
& +\sum_{j=1}^{n} \frac{\rho_{j}}{\rho_{i}}\left\{F_{i j}(\tau)\left[b_{i}(\tau)+C_{i j}(\tau)\right]+F_{i j}^{\prime}(\tau)\right\} u_{j}\left(\tau-\delta_{i j}(\tau)\right)+\frac{a_{i}(t)}{\rho_{i}}, \quad i=1,2, \cdots, n .
\end{aligned}
$$

Then, we have

Theorem 3.2 In addition to $\left(\mathrm{H}_{4}\right)-\left(\mathrm{H}_{6}\right)$, assume further that there exist positive constants $\rho_{i}(i=1,2, \cdots, n)$, such that

$\left(\mathrm{H}_{8}\right) \max _{t \in R} \int_{-\infty}^{t} \mathrm{e}^{-\int_{\tau}^{t}\left[b_{i}(\xi)+C_{i i}(\xi)\right] \mathrm{d} \xi} q_{i}(\tau) \mathrm{d} \tau<1-\left\|\sum_{j=1}^{n} \frac{\rho_{j}}{\rho_{i}} F_{i j}(t)\right\|_{1}$.

Then (1.5) has a unique positive almost periodic solution with strictly positive components, say

$$
N^{*}(t)=\left(N_{1}^{*}(t), N_{2}^{*}(t), \cdots, N_{n}^{*}(t)\right)^{\mathrm{T}},
$$

where

$$
q_{i}(\tau)=\sum_{j=1, j \neq i}^{n} \frac{\rho_{j}}{\rho_{i}} C_{i j}(\tau)+\sum_{j=1}^{n} \frac{\rho_{j}}{\rho_{i}}\left\{D_{i j}(\tau)+E_{i j}(\tau)+F_{i j}(\tau)\left[b_{i}(\tau)+C_{i i}(\tau)\right]+\left|F_{i j}^{\prime}(\tau)\right|\right\}
$$

and

$$
\left\|\sum_{j=1}^{n} \frac{\rho_{j}}{\rho_{i}} F_{i j}(t)\right\|_{1}=\max _{t \in[0, \omega]}\left\{\left|\sum_{j=1}^{n} \frac{\rho_{j}}{\rho_{i}} F_{i j}(t)\right|\right\} .
$$

$$
C=\left\{v(t)=\left(v_{1}(t), \cdots, v_{n}(t)\right)^{\mathrm{T}}\right.
$$

is continuous almost periodic function $\}$

Proof. Set

with the norm $\|v\|_{1}=\sup \left\{\|u(t)\|_{1}: t \in R\right\}$, obviously, $C$ is 
a Banach space. For any continuously almost periodic function $u(t)=\left(u_{1}(t), \cdots, u_{n}(t)\right)^{\mathrm{T}}$ we know that $x_{i u}(t)$ defined by (3.19) is also a continuously almost periodic function. Now define the mapping $\varphi: C \rightarrow C$ as follows:

$$
\varphi u(t)=x_{u}(t), u(t) \in C .
$$

Then similarly to the prove of Theorem 3.1 , we could prove that under the assumptions of Theorem 3.2, the mapping $\varphi$ is a contract mapping, and so system (3.19) has a unique fixed point $x(t)=\left(x_{1}(t), \cdots, x_{n}(t)\right)^{\mathrm{T}}$. and so,

$$
\begin{aligned}
& y(t)=\left(\exp \left\{\rho_{1} x_{1}(t)\right\}, \cdots, \exp \left\{\rho_{n} x_{n}(t)\right\}\right)^{\mathrm{T}} \quad \begin{array}{l}
\text { that there exist positive constants } \\
\text { such that }
\end{array} \\
& \qquad \begin{aligned}
q_{i}(t) & =\sum_{j=1, j \neq i}^{n} \frac{\rho_{j}}{\rho_{i}} C_{i j}(t)+\sum_{j=1}^{n} \frac{\rho_{j}}{\rho_{i}}\left\{D_{i j}(t)+E_{i j}(t)+F_{i j}(t)\left[b_{i}(t)+C_{i i}(t)\right]+\left|F_{i j}^{\prime}(t)\right|\right\} \\
& <\left[b_{i}(t)+C_{i i}(t)\right]\left(1-\left\|-\sum_{j=1}^{n} \frac{\rho_{j}}{\rho_{i}} F_{i j}(t)\right\|_{1}\right) .
\end{aligned}
\end{aligned}
$$

is the unique positive almost periodic solution of system (2.1), from Lemma 2.2,

$$
\begin{gathered}
N(t)=\left(\prod_{0<t_{k}<t}\left(1+\theta_{i k}\right) \exp \left\{\rho_{1} x_{1}(t)\right\}, \cdots,\right. \\
\left.\prod_{0<t_{k}<t}\left(1+\theta_{i k}\right) \exp \left\{\rho_{n} x_{n}(t)\right\}\right)^{\mathrm{T}}
\end{gathered}
$$

is the unique positive almost periodic solution of system (1.5). The proof is complete.

As a direct corollary of Theorem 3.2, one has

Corollary 3.2 In addition to $\left(\mathrm{H}_{4}\right)-\left(\mathrm{H}_{6}\right)$, assume further that there exist positive constants $\rho_{i}(i=1,2, \cdots, n)$,

Then (1.5) has a unique positive almost periodic solution with strictly positive components.

Consider the following equation:

$$
\begin{aligned}
\frac{\mathrm{d} N_{i}(t)}{\mathrm{d} t}= & N_{i}(t)\left[a_{i}(t)-b_{i}(t) \ln N_{i}(t)-\sum_{j=1}^{n} c_{i j}(t) \ln N_{j}(t)-\sum_{j=1}^{n} d_{i j}(t) \ln N_{j}\left(t-\gamma_{i j}(t)\right)\right. \\
& \left.-\sum_{j=1}^{n} e_{i j}(t) \int_{-\infty}^{t} K_{i j}(t-s) \ln N_{j}(s) \mathrm{d} s-\sum_{j=1}^{n} f_{i j}(t) \frac{d \ln N_{j}\left(t-\delta_{i j}(t)\right)}{d t}\right], \quad i=1,2, \ldots, n
\end{aligned}
$$

which is a special case of system (1.5) and (1.6) without impulse. Similarly, we can get the following results.

Theorem 3.3 In addition to $\left(\mathrm{H}_{1}\right)$, assume further that there exist positive constants $\rho_{i}(i=1,2, \cdots, n)$, such that

$\left(\mathrm{H}_{9}\right) \max _{t \in R} \int_{-\infty}^{t} \mathrm{e}^{-\int_{\tau}^{t}\left[b_{i}(\xi)+C_{i i}(\xi)\right] \mathrm{d} \xi} q_{i}(\tau) \mathrm{d} \tau<1-\left\|\sum_{j=1}^{n} \frac{\rho_{j}}{\rho_{i}} f_{i j}(t)\right\|_{1}$.

Then (1.5) has a unique positive $\omega$-periodic solution with strictly positive components, say

$$
N^{*}(t)=\left(N_{1}^{*}(t), N_{2}^{*}(t), \cdots, N_{n}^{*}(t)\right)^{\mathrm{T}} .
$$

where

$$
q_{i}(\tau)=\sum_{j=1, j \neq i}^{n} \frac{\rho_{j}}{\rho_{i}} c_{i j}(\tau)+\sum_{j=1}^{n} \frac{\rho_{j}}{\rho_{i}}\left\{d_{i j}(\tau)+e_{i j}(\tau)+f_{i j}(\tau)\left[b_{i}(\tau)+c_{i i}(\tau)\right]+\left|f_{i j}^{\prime}(\tau)\right|\right\}
$$

and

$$
\left\|\sum_{j=1}^{n} \frac{\rho_{j}}{\rho_{i}} f_{i j}(t)\right\|_{1}=\max _{t \in[0, \omega]}\left\{\left|\sum_{j=1}^{n} \frac{\rho_{j}}{\rho_{i}} f_{i j}(t)\right|\right\} .
$$

Proof. Similar to the proof of Theorem 3.1, we omit it here.

As a direct corollary of Theorem 3.3, one has

Corollary 3.3 In addition to $\left(\mathrm{H}_{1}\right)$, assume further that there exist positive constants $\rho_{i}(i=1,2, \cdots, n)$, such that

$$
\begin{aligned}
q_{i}(t) & =\sum_{j=1, j \neq i}^{n} \frac{\rho_{j}}{\rho_{i}} c_{i j}(t)+\sum_{j=1}^{n} \frac{\rho_{j}}{\rho_{i}}\left\{d_{i j}(t)+e_{i j}(t)+f_{i j}(t)\left[b_{i}(t)+c_{i i}(t)\right]+\left|f_{i j}^{\prime}(t)\right|\right\} \\
& <\left[b_{i}(t)+c_{i i}(t)\right]\left(1-\left\|\sum_{j=1}^{n} \frac{\rho_{j}}{\rho_{i}} f_{i j}(t)\right\|\right) .
\end{aligned}
$$


Then (1.5) has a unique positive $\omega$-periodic solution with strictly positive components.

Theorem 3.4 In addition to $\left(\mathrm{H}_{4}\right)$, assume further that there exist positive constants $\rho_{i}(i=1,2, \cdots, n)$, such that $\left(\mathrm{H}_{10}\right) \max _{t \in R} \int_{-\infty}^{t} \mathrm{e}^{-\int_{\tau}^{t}\left[b_{i}(\xi)+C_{i i}(\xi)\right] \mathrm{d} \xi} q_{i}(\tau) \mathrm{d} \tau<1-\left\|\sum_{j=1}^{n} \frac{\rho_{j}}{\rho_{i}} f_{i j}(t)\right\|_{1}$.

Then (1.5) has a unique positive almost periodic solution with strictly positive components, say

where

$$
N^{*}(t)=\left(N_{1}^{*}(t), N_{2}^{*}(t), \cdots, N_{n}^{*}(t)\right)^{\mathrm{T}} .
$$

$$
q_{i}(\tau)=\sum_{j=1, j \neq i}^{n} \frac{\rho_{j}}{\rho_{i}} c_{i j}(\tau)+\sum_{j=1}^{n} \frac{\rho_{j}}{\rho_{i}}\left\{d_{i j}(\tau)+e_{i j}(\tau)+f_{i j}(\tau)\left[b_{i}(\tau)+c_{i i}(\tau)\right]+\left|f_{i j}^{\prime}(\tau)\right|\right\}
$$

and

$$
\left\|\sum_{j=1}^{n} \frac{\rho_{j}}{\rho_{i}} f_{i j}(\tau)\right\|_{1}=\max _{t \in[0, \omega]}\left\{\left|\sum_{j=1}^{n} \frac{\rho_{j}}{\rho_{i}} f_{i j}(\tau)\right|\right\} .
$$

As a direct corollary of Theorem 3.4, one has

Corollary 3.4 In addition to $\left(\mathrm{H}_{4}\right)$, assume further that there exist positive constants $\rho_{i}(i=1,2, \cdots, n)$, such that

$$
\begin{aligned}
q_{i}(t) & =\sum_{j=1, j \neq i}^{n} \frac{\rho_{j}}{\rho_{i}} c_{i j}(t)+\sum_{j=1}^{n} \frac{\rho_{j}}{\rho_{i}}\left\{d_{i j}(t)+e_{i j}(t)+f_{i j}(t)\left[b_{i}(t)+c_{i i}(t)\right]+\left|f_{i j}^{\prime}(t)\right|\right\} \\
& <\left[b_{i}(t)+c_{i i}(t)\right]\left(1-\left|\sum_{j=1}^{n} \frac{\rho_{j}}{\rho_{i}} f_{i j}(t)\right|\right) .
\end{aligned}
$$

Then (1.5) has a unique positive almost periodic solution with strictly positive components.

\section{Global Asymptotic Stability}

In this section, we devote ourselves to the study of the global attractivity of periodic solutions (almost periodic solutions) of system (1.5), (1.6) and (3.22) (which is a special case of system (1.5) and (1.6) without impulse). Now, we state our main results of this section as follows:

Theorem 4.1. Assume that the conditions in Theorem 3.1 hold. Suppose further the following conditions hold:

$\left(\mathrm{H}_{11}\right)$ There is a positive constant $M$ such that

$$
\begin{aligned}
& \sum_{j=1}^{n}\left|\frac{\rho_{j}}{\rho_{i}} F_{i j}(t)\right|+\int_{0}^{t}\left\{\sum_{j=1}^{n}\left[\left|\frac{\rho_{j}}{\rho_{i}} H_{i j}(u)\right|+\left|\frac{\rho_{j}}{\rho_{i}} D_{i j}(u)\right|+\left|\frac{\rho_{j}}{\rho_{i}} E_{i j}(u)\right|\right]+\sum_{j=1, j \neq i}^{n}\left|\frac{\rho_{j}}{\rho_{i}} C_{i j}(u)\right|\right\} \mathrm{e}^{-\int_{u}^{t}\left[b_{i}(\xi)+C_{i i}(\xi)\right] \mathrm{d} \xi} \mathrm{d} u \leq M<1 ; \\
& \left(\mathrm{H}_{12}\right) \exp \left\{-\int_{0}^{t}\left(b_{i}(\xi)+C_{i i}(\xi)\right) \mathrm{d} \xi\right\} \rightarrow 0, \text { as } t \rightarrow+\infty, i=1,2, \cdots, n .
\end{aligned}
$$

Then system (1.5) and (1.6) has a unique periodic solution which is globally attractive.

Proof. Let $N^{*}(t)=\left(N_{1}^{*}(t), N_{2}^{*}(t), \cdots, N_{n}^{*}(t)\right)^{\mathrm{T}}$ be the unique positive periodic solution of system (1.5) and (1.6), whose existence and uniqueness are guarantee by Theorem 2.1, and $N(t)=\left(N_{1}(t), N_{2}(t), \cdots, N_{n}(t)\right)^{\mathrm{T}}$ be any other solution of system (1.5) and (1.6). Let

$$
N_{i}^{*}(t)=\prod_{0<t_{k}<t}\left(1+\theta_{i k}\right) \exp \left\{\rho_{i} x_{i}^{*}(t)\right\}, N_{i}(t)=\prod_{0<t_{k}<t}\left(1+\theta_{i k}\right) \exp \left\{\rho_{i} x_{i}(t)\right\}
$$

then, similar to Equation (3.1), we have

$$
\begin{aligned}
\frac{\mathrm{d} x_{i}^{*}(t)}{\mathrm{d} t}= & -\left[b_{i}(t)+C_{i i}(t)\right] x_{i}^{*}(t)-\sum_{j=1, j \neq i}^{n} \frac{\rho_{j}}{\rho_{i}} C_{i j}(t) x_{j}^{*}(t)-\sum_{j=1}^{n} \frac{\rho_{j}}{\rho_{i}} D_{i j}(t) x_{j}^{*}\left(t-\gamma_{i j}(t)\right) \\
& -\sum_{j=1}^{n} \frac{\rho_{j}}{\rho_{i}} E_{i j}(t) \int_{-\infty}^{t} K_{i j}(t-s) x_{j}^{*}(s) \mathrm{d} s-\sum_{j=1}^{n} \frac{\rho_{j}}{\rho_{i}} F_{i j}(t)\left(1-\delta_{i j}^{\prime}(t)\right)\left(x_{j}^{*}\left(t-\delta_{i j}(t)\right)\right)^{\prime}+\frac{a_{i}(t)}{\rho_{i}},
\end{aligned}
$$

and, 


$$
\begin{aligned}
\frac{\mathrm{d} x_{i}(t)}{\mathrm{d} t}= & -\left[b_{i}(t)+C_{i i}(t)\right] x_{i}(t)-\sum_{j=1, j \neq i}^{n} \frac{\rho_{j}}{\rho_{i}} C_{i j}(t) x_{j}(t)-\sum_{j=1}^{n} \frac{\rho_{j}}{\rho_{i}} D_{i j}(t) x_{j}\left(t-\gamma_{i j}(t)\right) \\
& -\sum_{j=1}^{n} \frac{\rho_{j}}{\rho_{i}} E_{i j}(t) \int_{-\infty}^{t} K_{i j}(t-s) x_{j}(s) \mathrm{d} s-\sum_{j=1}^{n} \frac{\rho_{j}}{\rho_{i}} F_{i j}(t)\left(1-\delta_{i j}^{\prime}(t)\right) x_{j}^{\prime}\left(t-\delta_{i j}(t)\right)+\frac{a_{i}(t)}{\rho_{i}},
\end{aligned}
$$

Then, from (4.1) and (4.2), we have

$$
\begin{aligned}
\frac{\mathrm{d} x_{i}^{*}(t)}{\mathrm{d} t}-\frac{\mathrm{d} x_{i}(t)}{\mathrm{d} t}= & -\left[b_{i}(t)+C_{i i}(t)\right]\left(x_{i}^{*}(t)-x_{i}(t)\right)-\sum_{j=1, j \neq i}^{n} \frac{\rho_{j}}{\rho_{i}} C_{i j}(t)\left(x_{j}^{*}(t)-x_{j}(t)\right) \\
& -\sum_{j=1}^{n} \frac{\rho_{j}}{\rho_{i}} D_{i j}(t)\left(x_{j}^{*}\left(t-\gamma_{i j}(t)\right)-x_{j}\left(t-\gamma_{i j}(t)\right)\right) \\
& -\sum_{j=1}^{n} \frac{\rho_{j}}{\rho_{i}} E_{i j}(t) \int_{-\infty}^{t} K_{i j}(t-s)\left(x_{j}^{*}(s)-x_{j}(s)\right) \mathrm{d} s \\
& -\sum_{j=1}^{n} \frac{\rho_{j}}{\rho_{i}} F_{i j}(t)\left(1-\delta_{i j}^{\prime}(t)\right)\left(x_{j}^{*}\left(t-\delta_{i j}(t)\right)-x_{j}\left(t-\delta_{i j}(t)\right)\right)^{\prime}, \quad i=1,2, \cdots, n .
\end{aligned}
$$

Let $x_{i}^{*}(t)-x_{i}(t)=z_{i}(t)$, then

$$
\begin{aligned}
\frac{\mathrm{d} z_{i}(t)}{\mathrm{d} t}= & -\left[b_{i}(t)+C_{i i}(t)\right] z_{i}(t)-\sum_{j=1, j \neq i}^{n} \frac{\rho_{j}}{\rho_{i}} C_{i j}(t) z_{j}(t)-\sum_{j=1}^{n} \frac{\rho_{j}}{\rho_{i}} D_{i j}(t) z_{j}\left(t-\gamma_{i j}(t)\right) \\
& -\sum_{j=1}^{n} \frac{\rho_{j}}{\rho_{i}} E_{i j}(t) \int_{-\infty}^{t} K_{i j}(t-s) z_{j}(s) \mathrm{d} s-\sum_{j=1}^{n} \frac{\rho_{j}}{\rho_{i}} F_{i j}(t)\left(1-\delta_{i j}^{\prime}(t)\right) z_{j}^{\prime}\left(t-\delta_{i j}(t)\right), i=1,2, \cdots, n .
\end{aligned}
$$

Multiply both sides of (4.4) with $\exp \left\{\int_{0}^{t}\left(b_{i}(\xi)+C_{i i}(\xi)\right) \mathrm{d} \xi\right\}$ and then integrate from 0 to $t$ to obtain

$$
\begin{aligned}
& \int_{0}^{t}\left[z_{i}(u) \exp \left\{\int_{0}^{u}\left(b_{i}(\xi)+C_{i i}(\xi)\right) \mathrm{d} \xi\right\}\right]^{\prime} \mathrm{d} u \\
& =-\int_{0}^{t}\left[\sum_{j=1, j \neq i}^{n} \frac{\rho_{j}}{\rho_{i}} C_{i j}(u) z_{j}(u)+\sum_{j=1}^{n}\left(\frac{\rho_{j}}{\rho_{i}} D_{i j}(u) z_{j}\left(u-\gamma_{i j}(u)\right)+\frac{\rho_{j}}{\rho_{i}} E_{i j}(u) \int_{-\infty}^{u} K_{i j}(u-s) z_{j}(s) \mathrm{d} s\right.\right. \\
& \left.\left.\quad+\frac{\rho_{j}}{\rho_{i}} F_{i j}(u)\left(1-\delta_{i j}^{\prime}(u)\right) z_{j}^{\prime}\left(u-\delta_{i j}(u)\right)\right)\right] \exp \left\{\int_{0}^{u}\left(b_{i}(\xi)+C_{i i}(\xi)\right) \mathrm{d} \xi\right\} \mathrm{d} u, i=1,2, \cdots, n,
\end{aligned}
$$

then

$$
\begin{aligned}
& z_{i}(t) \exp \left\{\int_{0}^{t}\left(b_{i}(\xi)+C_{i i}(\xi)\right) \mathrm{d} \xi\right\} \\
& =z_{i}(0)-\int_{0}^{t}\left[\sum_{j=1, j \neq i}^{n} \frac{\rho_{j}}{\rho_{i}} C_{i j}(u) z_{j}(u)+\sum_{j=1}^{n}\left(\frac{\rho_{j}}{\rho_{i}} D_{i j}(u) z_{j}\left(u-\gamma_{i j}(u)\right)+\frac{\rho_{j}}{\rho_{i}} E_{i j}(u) \int_{-\infty}^{u} K_{i j}(u-s) z_{j}(s) \mathrm{d} s\right.\right. \\
& \left.\left.\quad+\frac{\rho_{j}}{\rho_{i}} F_{i j}(u)\left(1-\delta_{i j}^{\prime}(u)\right) z_{j}^{\prime}\left(u-\delta_{i j}(u)\right)\right)\right] \exp \left\{\int_{0}^{u}\left(b_{i}(\xi)+C_{i i}(\xi)\right) \mathrm{d} \xi\right\} \mathrm{d} u, i=1,2, \cdots, n,
\end{aligned}
$$

thus

$$
\begin{aligned}
z_{i}(t)= & z_{i}(0) \exp \left\{-\int_{0}^{t}\left(b_{i}(\xi)+C_{i i}(\xi)\right) \mathrm{d} \xi\right\} \\
& -\int_{0}^{t}\left[\sum_{j=1, j \neq i}^{n} \frac{\rho_{j}}{\rho_{i}} C_{i j}(u) z_{j}(u)+\sum_{j=1}^{n}\left(\frac{\rho_{j}}{\rho_{i}} D_{i j}(u) z_{j}\left(u-\gamma_{i j}(u)\right)+\frac{\rho_{j}}{\rho_{i}} E_{i j}(u) \int_{-\infty}^{u} K_{i j}(u-s) z_{j}(s) \mathrm{d} s\right.\right. \\
& \left.\left.+\frac{\rho_{j}}{\rho_{i}} F_{i j}(u)\left(1-\delta_{i j}^{\prime}(u)\right) z_{j}^{\prime}\left(u-\delta_{i j}(u)\right)\right)\right] \exp \left\{-\int_{u}^{t}\left(b_{i}(\xi)+C_{i i}(\xi)\right) \mathrm{d} \xi\right\} \mathrm{d} u, i=1,2, \cdots, n .
\end{aligned}
$$


Let $F_{i j}^{*}(u)=F_{i j}(u)\left(1-\delta_{i j}^{\prime}(u)\right)$, by Lemma 2.3, we obtain

$$
\begin{aligned}
& \int_{0}^{t} \sum_{j=1}^{n} \frac{\rho_{j}}{\rho_{i}} F_{i j}(t)\left(1-\delta_{i j}^{\prime}(t)\right) z_{j}^{\prime}\left(t-\delta_{i j}(t)\right) \exp \left\{-\int_{u}^{t}\left(b_{i}(\xi)+C_{i i}(\xi)\right) \mathrm{d} \xi\right\} \mathrm{d} u \\
&=\int_{0}^{t} \sum_{j=1}^{n} \frac{\rho_{j}}{\rho_{i}} F_{i j}^{*}(u) z_{j}^{\prime}\left(u-\delta_{i j}(u)\right) \exp \left\{-\int_{u}^{t}\left(b_{i}(\xi)+C_{i i}(\xi)\right) \mathrm{d} \xi\right\} \mathrm{d} u \\
&=\int_{0}^{t} \sum_{j=1}^{n} \frac{F_{i j}^{*}(u) z_{j}^{\prime}\left(u-\delta_{i j}(u)\right)\left(1-\delta_{i j}^{\prime}(u)\right)}{1-\delta_{i j}^{\prime}(u)} \exp \left\{-\int_{u}^{t}\left(b_{i}(\xi)+C_{i i}(\xi)\right) \mathrm{d} \xi\right\} \mathrm{d} u \\
&=\int_{0}^{t} \sum_{j=1}^{n} \frac{\rho_{j}}{\rho_{i}}\left[\frac{F_{i j}^{*}(u) \exp \left\{-\int_{u}^{t}\left(b_{i}(\xi)+C_{i i}(\xi)\right) \mathrm{d} \xi\right\}}{1-\delta_{i j}^{\prime}(u)}\right]\left[z_{i}^{\prime}\left(u-\delta_{i j}(u)\right)\left(1-\delta_{i j}^{\prime}(u)\right)\right] \mathrm{d} u \\
&=\sum_{j=1}^{n} \frac{\rho_{j}}{\rho_{i}}\left[\frac{F_{i j}^{*}(t)}{1-\delta_{i j}^{\prime}(t)} z_{j}\left(t-\delta_{i j}(t)\right)-\frac{F_{i j}^{*}(0)}{1-\delta_{i j}^{\prime}(0)} z_{j}\left(-\delta_{i j}(0)\right) \exp \left\{-\int_{u}^{t}\left(b_{i}(\xi)+C_{i i}(\xi)\right) \mathrm{d} \xi\right\}\right. \\
&\left.-\int_{0}^{t} H_{i j}(u) \exp \left\{-\int_{u}^{t}\left(b_{i}(\xi)+C_{i i}(\xi)\right) \mathrm{d} \xi\right\} z_{j}\left(u-\delta_{i j}(u)\right) \mathrm{d} u\right] \\
&= \sum_{j=1}^{n} \frac{\rho_{j}}{\rho_{i}}\left[F_{i j}(t) z_{j}\left(t-\delta_{i j}(t)\right)-F_{i j}(0) z_{j}\left(-\delta_{i j}(0)\right) \exp \left\{-\int_{u}^{t}\left(b_{i}(\xi)+C_{i i}(\xi)\right) \mathrm{d} \xi\right\}\right. \\
&\left.-\int_{0}^{t} H_{i j}(u) \exp \left\{-\int_{u}^{t}\left(b_{i}(\xi)+C_{i i}(\xi)\right) \mathrm{d} \xi\right\} z_{j}\left(u-\delta_{i j}(u)\right) \mathrm{d} u\right]
\end{aligned}
$$

where

$$
H_{i j}(u)=\frac{\left[F_{i j}^{\prime}(u)+F_{i j}(u)\left(b_{i}(u)+C_{i i}(u)\right)\right]\left(\left(1-\delta_{i j}^{\prime}(u)\right)+D_{i j}(u) \delta_{i j}^{\prime \prime}(u)\right)}{\left(1-\delta_{i j}^{\prime}(u)\right)^{2}} .
$$

Thus,

$$
\begin{aligned}
z_{i}(t)= & {\left[z_{i}(0)+\frac{\rho_{j}}{\rho_{i}} F_{i j}(0) z_{j}\left(-\delta_{i j}(0)\right)\right] \exp \left\{-\int_{0}^{t}\left(b_{i}(\xi)+C_{i i}(\xi)\right) \mathrm{d} \xi\right\} } \\
& +\int_{0}^{t}\left\{\sum_{j=1}^{n}\left[\frac{\rho_{j}}{\rho_{i}} H_{i j}(u) z_{j}\left(u-\delta_{i j}(u)\right)-\frac{\rho_{j}}{\rho_{i}} D_{i j}(t) z_{j}\left(t-\gamma_{i j}(t)\right)-\frac{\rho_{j}}{\rho_{i}} E_{i j}(t) \int_{-\infty}^{t} K_{i j}(t-s) z_{j}(s) \mathrm{d} s\right]\right. \\
& \left.-\sum_{j=1, j \neq i}^{n} \frac{\rho_{j}}{\rho_{i}} C_{i j}(u) z_{j}(u)\right\} \exp \left\{-\int_{u}^{t}\left(b_{i}(\xi)+C_{i i}(\xi)\right) \mathrm{d} \xi\right\} \mathrm{d} u-\sum_{j=1}^{n} \frac{\rho_{j}}{\rho_{i}} F_{i j}(t) z_{j}\left(t-\delta_{i j}(t)\right), i=1,2, \cdots, n,
\end{aligned}
$$

then

$$
\begin{aligned}
\left|z_{i}\right| \leq & \left|z_{i}(0)+\frac{\rho_{j}}{\rho_{i}} F_{i j}(0) z_{j}\left(-\delta_{i j}(0)\right)\right| \exp \left\{-\int_{0}^{t}\left(b_{i}(\xi)+C_{i i}(\xi)\right) \mathrm{d} \xi\right\} \\
& +\int_{0}^{t}\left\{\sum_{j=1}^{n}\left[\left|\frac{\rho_{j}}{\rho_{i}} H_{i j}(u)\right|+\left|\frac{\rho_{j}}{\rho_{i}} D_{i j}(u)\right|+\left|\frac{\rho_{j}}{\rho_{i}} E_{i j}(u)\right|\right]+\sum_{j=1, j \neq i}^{n}\left|\frac{\rho_{j}}{\rho_{i}} C_{i j}(u)\right|\right\} \exp \left\{-\int_{u}^{t}\left(b_{i}(\xi)+C_{i i}(\xi)\right) \mathrm{d} \xi\right\} \mathrm{d} u\left|z_{j}\right| \\
& +\sum_{j=1}^{n}\left|\frac{\rho_{j}}{\rho_{i}} F_{i j}(t)\right|\left|z_{j}\right|, i=1,2, \cdots, n,
\end{aligned}
$$

That is 


$$
\begin{aligned}
\|z\|_{1} \leq & \left|z_{i}(0)+\frac{\rho_{j}}{\rho_{i}} F_{i j}(0) z_{j}\left(-\delta_{i j}(0)\right)\right| \exp \left\{-\int_{0}^{t}\left(b_{i}(\xi)+C_{i i}(\xi)\right) \mathrm{d} \xi\right\} \\
& +\int_{0}^{t}\left\{\sum_{j=1}^{n}\left[\left|\frac{\rho_{j}}{\rho_{i}} H_{i j}(u)\right|+\left|\frac{\rho_{j}}{\rho_{i}} D_{i j}(u)\right|+\left|\frac{\rho_{j}}{\rho_{i}} E_{i j}(u)\right|\right]+\sum_{j=1, j \neq i}^{n}\left|\frac{\rho_{j}}{\rho_{i}} C_{i j}(u)\right|\right\} \exp \left\{-\int_{u}^{t}\left(b_{i}(\xi)+C_{i i}(\xi)\right) \mathrm{d} \xi\right\} \mathrm{d} u\|z\|_{1} \\
& +\sum_{j=1}^{n}\left|\frac{\rho_{j}}{\rho_{i}} F_{i j}(t)\right|\|z\|_{1}, i=1,2, \cdots, n .
\end{aligned}
$$

From $\left(\mathrm{H}_{3}\right)$, we have

$$
\begin{aligned}
\|w\|_{1} \leq & \frac{\left|z_{i}(0)+\frac{\rho_{j}}{\rho_{i}} F_{i j}(0) z_{j}\left(-\delta_{i j}(0)\right)\right| \mathrm{e}^{-\int_{0}^{t}\left(b_{i}(\xi)+C_{i i}(\xi)\right) \mathrm{d} \xi}}{1-\sum_{j=1}^{n}\left|\frac{\rho_{j}}{\rho_{i}} F_{i j}(t)\right|-\int_{0}^{t}\left\{\sum_{j=1}^{n}\left[\left|\frac{\rho_{j}}{\rho_{i}} H_{i j}(u)\right|+\left|\frac{\rho_{j}}{\rho_{i}} D_{i j}(u)\right|+\left|\frac{\rho_{j}}{\rho_{i}} E_{i j}(u)\right|\right]+\sum_{j=1, j \neq i}^{n}\left|\frac{\rho_{j}}{\rho_{i}} C_{i j}(u)\right|\right\} \mathrm{e}^{-\int_{u}^{t}\left(b_{i}(\xi)+C_{i i}(\xi)\right) \mathrm{d} \xi} \mathrm{d} u} \\
& \leq \frac{\left|z_{i}(0)+\frac{\rho_{j}}{\rho_{i}} F_{i j}(0) z_{j}\left(-\delta_{i j}(0)\right)\right| \mathrm{e}^{-\int_{0}^{t}\left(b_{i}(\xi)+C_{i i}(\xi)\right) \mathrm{d} \xi}}{1-M}, \quad i=1,2, \cdots, n .
\end{aligned}
$$

From $\left(\mathrm{H}_{4}\right)$, we have

$$
\|z\|_{1}=\max _{t \in[0, \omega]}\left|z_{i}(t)\right|=\max _{t \in[0, \omega]}\left|x_{i}^{*}(t)-x_{i}(t)\right|=0, \text { as } t \rightarrow+\infty, i=1,2, \cdots, n,
$$

thus, $x_{i}(t) \rightarrow x_{i}^{*}(t)$, as $t \rightarrow+\infty, i=1,2, \cdots, n$, that is the positive $\omega$-periodic solution of (3.1) is globally attractive,

$$
\begin{aligned}
N_{i}(t) & =\prod_{0<t_{k}<t}\left(1+\theta_{i k}\right) \exp \left\{\rho_{i} x_{i}(t)\right\} \\
& \rightarrow \prod_{0<t_{k}<t}\left(1+\theta_{i k}\right) \exp \left\{\rho_{i} x_{i}^{*}(t)\right\}=N_{i}^{*}(t) \text { as } t \rightarrow+\infty, i=1,2, \cdots, n,
\end{aligned}
$$

by Definition 2.2, the positive $\omega$-periodic solution of (1.5) is globally attractive. The proof is completed.

Theorem 4.2. Assume that the conditions in Theorem 3.2 hold. Suppose further the following conditions hold:

$\left(\mathrm{H}_{13}\right)$ There is a positive constant $\mathrm{m}$ such that

$$
\sum_{j=1}^{n}\left|\frac{\rho_{j}}{\rho_{i}} F_{i j}(t)\right|+\int_{0}^{t}\left\{\sum_{j=1}^{n}\left[\left|\frac{\rho_{j}}{\rho_{i}} H_{i j}(u)\right|+\left|\frac{\rho_{j}}{\rho_{i}} D_{i j}(u)\right|+\left|\frac{\rho_{j}}{\rho_{i}} E_{i j}(u)\right|\right]+\sum_{j=1, j \neq i}^{n}\left|\frac{\rho_{j}}{\rho_{i}} C_{i j}(u)\right|\right\} \mathrm{e}^{-\int_{u}^{t}\left(b_{i}(\xi)+C_{i i}(\xi)\right) \mathrm{d} \xi} \mathrm{d} u \leq m<1 ;
$$

$\left(\mathrm{H}_{14}\right) \exp \left\{-\int_{0}^{t}\left(b_{i}(\xi)+C_{i i}(\xi)\right) \mathrm{d} \xi\right\} \rightarrow 0$, as $t \rightarrow+\infty, i=1,2, \cdots, n$.

Then system (1.5) and (1.6) has a unique almost periodic solution which is globally attractive.

Proof. Similar to the proof of Theorem 4.1, we omit it here.

Theorem 4.3. Assume that the conditions in Theorem 3.3 (or Theorem 3.4) hold. Suppose further the following conditions hold:

$\left(\mathrm{H}_{15}\right)$ There is a positive constant $\lambda$ such that

$$
\sum_{j=1}^{n}\left|\frac{\rho_{j}}{\rho_{i}} f_{i j}(t)\right|+\int_{0}^{t}\left\{\sum_{j=1}^{n}\left[\left|\frac{\rho_{j}}{\rho_{i}} H_{i j}^{*}(u)\right|+\left|\frac{\rho_{j}}{\rho_{i}} d_{i j}(u)\right|+\left|\frac{\rho_{j}}{\rho_{i}} e_{i j}(u)\right|\right]+\sum_{j=1, j \neq i}^{n}\left|\frac{\rho_{j}}{\rho_{i}} c_{i j}(u)\right|\right\} \mathrm{e}^{-\int_{u}^{t}\left(b_{i}(\xi)+C_{i i}(\xi)\right) \mathrm{d} \xi} \mathrm{d} u \leq \lambda<1 ;
$$

$\left(\mathrm{H}_{16}\right) \exp \left\{-\int_{0}^{t}\left(b_{i}(\xi)+c_{i i}(\xi)\right) \mathrm{d} \xi\right\} \rightarrow 0$, as $t \rightarrow+\infty, i=1,2, \cdots, n$.

Then system (3.22) has a unique periodic solution (almost periodic solution) which is globally attractive, where

$$
H_{i j}^{*}(u)=\frac{\left[f_{i j}^{\prime}(u)+f_{i j}(u)\left(b_{i}(u)+c_{i i}(u)\right)\right]\left(\left(1-\delta_{i j}^{\prime}(u)\right)+d_{i j}(u) \delta_{i j}^{\prime \prime}(u)\right)}{\left(1-\delta_{i j}^{\prime}(u)\right)^{2}} .
$$

Proof. Similar to the proof of Theorem 4.1, we omit it here. 


\section{An Example}

Now, we give an example to demonstrate our result. Let us consider the following equation:

$$
\begin{aligned}
\frac{\mathrm{d} N(t)}{\mathrm{d} t}= & N(t)\left[2+\cos t-\frac{1}{4} \ln N(t)-\frac{2+\sin t}{20} \ln N(t-2 \pi-0.01-0.5 \sin t)\right. \\
& \left.-\frac{1-\sin t}{20} \int_{-\infty}^{t} K_{j}(t-s) \ln N(s) \mathrm{d} s-\frac{1}{10} \ln N(t-0.1+0.5 \sin t)\right]
\end{aligned}
$$

Compare with (3.22), we get $i=j=1$,

$$
\begin{gathered}
a_{1}(t)=2+\cos t, \quad b_{1}(t)=\frac{1}{4}, \quad c_{11}(t)=0, \\
\begin{aligned}
& d_{11}(t)=\frac{2+\sin t}{20}, \quad e_{11}(t)=\frac{1-\sin t}{20}, \quad f_{11}(t)=\frac{1}{10}, \\
& \gamma_{11}(t)=2 \pi+0.01+0.5 \sin t, \quad \delta_{11}(t)=0.1-0.5 \sin t . \\
& \text { So, } \gamma_{11}^{\prime}(t)=0.5 \cos t<1, \quad \delta_{11}^{\prime}(t)=-0.5 \cos t<1, \\
& \delta_{11}^{\prime \prime}(t)=0.5 \sin t, \quad f_{11}(t)=0 \text { and } \\
& q_{1}(t)=d_{11}(t)+e_{11}(t)+f_{11}(t) b_{1}(t)+\left|f_{11}^{\prime}(t)\right| \\
&=\frac{2+\sin t}{20}+\frac{1-\sin t}{20}+\frac{1}{10} \times \frac{1}{4} \\
&=\frac{7}{40}<b_{1}(t)\left(1-\left\|f_{11}(t)\right\|_{1}\right)=\frac{9}{40} .
\end{aligned}
\end{gathered}
$$

According to Corollary 3.3, we see that system (5.1) has at least one positive $2 \pi$-periodic solution.

\section{Acknowledgements}

This work was supported by the construct program of the key discipline in Hunan province.

\section{REFERENCES}

[1] Q. Wang, Y. Wang and B. X. Dai, "Existence and Uniqueness of Positive Periodic Solutions for a Neutral Logarithmic Population Model," Applied Mathematics and Computation, Vol. 213, No. 1, 2009, pp. 137-147. doi:10.1016/j.amc.2009.03.028

[2] L. Berezansky and E. Braverman, "Explicit Conditions of Exponential Stability for a Linear Impulsive Delay Differential Equation," Journal of Mathematical Analysis and Applications, Vol. 214, No. 2, 1997, pp. 439-458. doi:10.1006/jmaa.1997.5578

[3] H. Fang, "Positive Periodic Solutions of $N$-Species Neutral Delay Systems," Czechoslovak Mathematical Journal, Vol. 53, No. 3, 2003, pp. 561-570. doi:10.1023/B:CMAJ.0000024503.03321.b1

[4] F. D. Chen, "On a Nonlinear Nonautonomous PredatorPrey Model with Diffusion and Distributed Delay," Journal of Computational and Applied Mathematics, Vol. 180, No. 1, 2005, pp. 33-49. doi:10.1016/i.cam.2004.10.001

[5] H. Fang and J. B. Li, "On the Existence of Periodic Solutions of a Neutral Delay Model of Single-Species Population Growth," Journal of Mathematical Analysis and Ap- plications, Vol. 259, No. 1, 2001, pp. 8-17. doi:10.1006/jmaa.2000.7340

[6] F. D. Chen, "Periodic Solutions and Almost Periodic Solutions for a Delay Multispecies Logarithmic Population Model," Applied Mathematics and Computation, Vol. 171, No. 2, 2005, pp. 760-770. doi:10.1016/j.amc.2005.01.085

[7] Z. H. Yang and J. D. Cao, "Positive Periodic Solutions of Neutral Lotka-Volterra System with Periodic Delays," Applied Mathematics and Computation, Vol. 149, No. 3, 2004, pp. 661-687. doi:10.1016/S0096-3003(03)00170-X

[8] Y. K. Li, “On a Periodic Neutral Delay Logarithmic Population Model," Journal of Systems Science and Complexity, Vol. 19, No. 1, 1999, pp. 34-38. (in Chinese)

[9] S. P. Lu and W. G. Ge, "Existence of Positive Periodic Solutions for Neutral Logarithmic Population Model with Multiple Delays," Journal of Computational and Applied Mathematics, Vol. 166, No. 2, 2004, pp. 371-383. doi:10.1016/j.cam.2003.08.033

[10] F. D. Chen, "Periodic Solutions and Almost Periodic Solutions of a Neutral Multispecies Logarithmic Population Model," Applied Mathematics and Computation, Vol. 176, No. 2, 2006, pp. 431-441. doi:10.1016/j.amc.2005.09.032

[11] R. E. Gaines and J. L. Mawkin, "Coincidence Degree and Nonlinear Differential Equations," Springer-Verlag, Berlin, 1977.

[12] X. Z. Liu and G. Ballinger, "Existence and Continuability of Solutions for Differential Equations with Delay and State-Dependent Impulses," Nonlinear Analysis: Theory, Methods \& Applications, Vol. 51, No. 4, 2002, pp. 633647. doi:10.1016/S0362-546X(01)00847-1

[13] B. G. Zhang and Y. J. Liu, "Global Attractivity for Certain Impulsive Delay Differential Equations," Nonlinear Analysis: Theory, Methods \& Applications, Vol. 52, No. 3, 2003, pp. 725-736. doi:10.1016/S0362-546X(02)00129-3

[14] Y. J. Zhang, B. Liu and L. S. Chen, "Extinction and Permanence of a Two-Prey One-Predator System with Impulsive Effect," Mathematical Medicine and Biology, Vol. 20, No. 4, 2003, pp. 309-325. doi:10.1093/imammb/20.4.309

[15] J. R. Yan, "Existence of Positive Periodic Solutions of Impulsive Functional Differential Equations with Two Parameters," Journal of Mathematical Analysis and Applications, Vol. 327, No. 2, 2007, pp. 854-868. doi:10.1016/j.jmaa.2006.04.018

[16] W. M. Wang, H. L. Wang and Z. Q. Li, "Chaotic Behavior of a Three-Species Beddington-Type System with Impulsive Peturbations," Chaos, Solitons \& Fractals, Vol. 
37, No. 2, 2008, pp. 438-443. doi:10.1016/j.chaos.2006.09.013

[17] J. S. Yu, "Explicit Conditions for Stability of Nonlinear Scalar Delay Differential Equations with Impulses," Nonlinear Analysis: Theory, Methods \& Applications, Vol. 46, No. 1, 2001, pp. 53-67. doi:10.1016/S0362-546X(99)00445-9

[18] X. N. Liu and L. S. Chen, "Complex Dynamics of Holling Type II Lotka-Volterra Predator-Prey System with Impulsive Perturbations on the Predator," Chaos, Solitons \& Fractals, Vol. 16, No. 2, 2003, pp. 311-320. doi:10.1016/S0960-0779(02)00408-3

[19] Y. J. Liu and W. G. Ge, "Stability Theorems and Existence Results for Periodic Solutions of Nonlinear Impulsive Delay Differential Equations with Variable Coefficients," Nonlinear Analysis: Theory, Methods \& Applications, Vol. 57, No. 3, 2004, pp. 363-399. doi:10.1016/j.na.2004.02.020

[20] J. R. Yan and A. M. Zhao, "Oscillation and Stability of Linear Impulsive Delay Differential Equations," Journal of Mathematical Analysis and Applications, Vol. 227, No. 1, 1998, pp. 187-194. doi:10.1006/jmaa.1998.6093

[21] V. Lakshmikantham, D. Bainov and P. Simeonov, "Theory of Impulsive Differential Equations," World Scientific Publisher, Singapore City, 1989.

[22] A. M. Samoikleno and N. A. Perestyuk, "Impulsive Differential Equations," World Scientific, Singapore City, 1995.

[23] J. J. Nieto and R. Rodriguez-Lopez, "Periodic Boundary Value Problem for Non-Lipschitzian Impulsive Functional Differential Equations," Journal of Mathematical Analysis and Applications, Vol. 318, No. 2, 2006, pp.
593-610. doi:10.1016/i.jmaa.2005.06.014

[24] H. F. Huo, "Existence of Positive Periodic Solutions of a Neutral Delay Lotka-Volterra Systems with Impulses," Computers \& Mathematics with Applications, Vol. 48, No. 12, 2004, pp. 1833-1846. doi:10.1016/j.camwa.2004.07.009

[25] A. Ouahab, "Existence and Uniqueness Results for Impulsive Functional Differential Equations with Scalar Multiple Delay and Infinite Delay," Nonlinear Analysis: Theory, Methods \& Applications, Vol. 67, No. 4, 2007, pp. 1027-1041. doi:10.1016/j.na.2006.06.033

[26] F. D. Chen, "On the Existence and Uniqueness of Periodic Solutions of a Kind of Integro-Differential Equations," Acta Mathematica Sinica, Vol. 47, 2004, pp. 973 985. (in Chinese)

[27] H. Q. Xie and Q. Y. Wang, "Exponential Stability and Periodic Solution for Cellular Neural Networks with Time Delay," Journal of Huaqiao University, Vol. 25, 2004, pp. 22-26. (in Chinese)

[28] S. P. Lu and W. G. Ge, "Existence of Positive Periodic Solutions for Neutral Population Model with Multiple Delays," Applied Mathematics and Computation, Vol. 153, No. 3, 2004, pp. 885-892. doi:10.1016/S0096-3003(03)00685-4

[29] Y. H. Xia, "Positive Periodic Solutions for a Neutral Impulsive Delayed Lotka-Volterra Competition Systems with the Effect of Toxic Substance," Nonlinear Analysis: Real World Applications, Vol. 8, No. 1, 2007, pp. 204-221. doi:10.1016/j.nonrwa.2005.07.002

[30] L. Barbalat, "Systemes d'Equations Differentielles d'Oscillations Nonlineaires," Revue Roumaine de Mathématique Pures et Appliquées, Vol. 4, 1959, pp. 267-270. 